\section{Author's Accepted Manuscript}

Direct numerical simulations of flow in realistic mouth-throat geometries

L. Nicolaou, T.A. Zaki

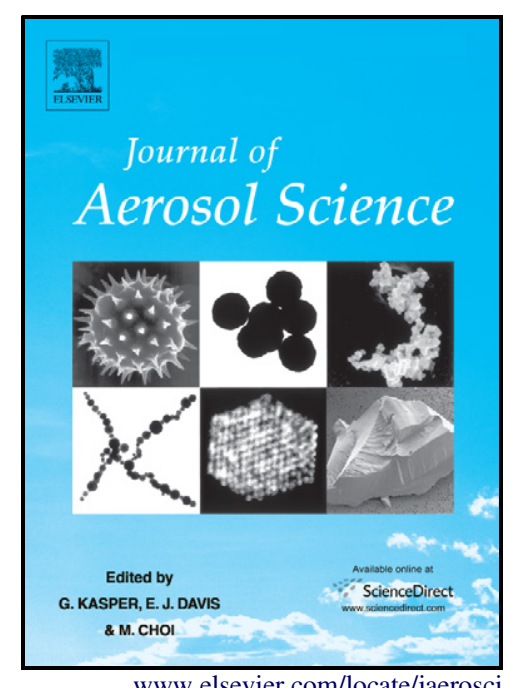

www.elsevier.com/locate/jaerosci

PII: S0021-8502(12)00171-1

DOI: http://dx.doi.org/10.1016/j.jaerosci.2012.10.003

Reference: AS4598

To appear in: Journal of Aerosol Science

Received date: 18 May 2012

Revised date: 9 October 2012

Accepted date: 10 October 2012

Cite this article as: L. Nicolaou and T.A. Zaki, Direct numerical simulations of flow in realistic mouth-throat geometries, Journal of Aerosol Science, http://dx.doi.org/10.1016/ j.jaerosci.2012.10.003

This is a PDF file of an unedited manuscript that has been accepted for publication. As a service to our customers we are providing this early version of the manuscript. The manuscript will undergo copyediting, typesetting, and review of the resulting galley proof before it is published in its final citable form. Please note that during the production process errors may be discovered which could affect the content, and all legal disclaimers that apply to the journal pertain. 


\title{
Direct numerical simulations of flow in realistic mouth-throat geometries
}

\author{
L. Nicolaou, T. A. Zaki* \\ Department of Mechanical Engineering, Imperial College London, \\ Exhibition Road, London SW7 2AZ, UK
}

\begin{abstract}
The flow in a set of four realistic mouth-throat geometries at a flow rate of $30 \mathrm{~L} / \mathrm{min}$ is studied in order to determine the effect of intrasubject and intersubject variation on the mean flow patterns and the turbulence fluctuations. Direct numerical simulations (DNS) are performed, which fully resolve all the scales in the flow, without requiring a turbulence model. An immersed boundary method is applied on curvilinear grids which simplifies the task of grid generation for the complex extrathoracic geometries and allows the use of a structured grid solver which increases the efficiency of the numerical scheme. Inspection of the mean, responsible for the convective transport of particles, and the fluctuating component of velocity, responsible for turbulent dispersion, allows us to explain in vitro deposition data in the literature obtained in the same mouth-throat models. The results provide insight as to how geometric variation affects aerosol deposition and explain the scatter in deposition data observed in the literature. Geometric variation is shown to have a large impact on both the mean velocity profiles and the turbulence intensities. Examination of the flow fields in the various mouth-throat geometries allows us to address the origin of the dependence of deposition on Reynolds number, and provide the physical significance of the empirical Reynolds number correction previously proposed in the literature.
\end{abstract}

Keywords: intersubject, intrasubject, realistic mouth-throat geometry, Reynolds number effect, DNS

*Corresponding author. Tel.: +44(0)2075947032; fax: +44(0)2075945702.

Email address: t.zaki@imperial.ac.uk (T. A. Zaki) 


\section{Introduction}

Aerosolized delivery of drugs to the lungs has been used for decades to treat a number of respiratory diseases such as asthma, chronic obstructive pulmonary disease (COPD), cystic fibrosis and pulmonary infections. The aerosol is generally inhaled through the mouth as it is more effective than the nasal route, allowing a higher dose to penetrate through the throat and into the lungs. For effective drug delivery, the aerosol must reach the target site within the lung. Often however, significant losses are experienced in the extrathoracic airways leading to very low pulmonary deposition. Aerosol deposition is highly dependent on the flow in the extrathoracic airways and therefore, understanding the flow dynamics in this region is important, in order to minimize extrathoracic losses and optimize pulmonary drug delivery. To this end, direct numerical simulations (DNS) have been performed in realistic mouth-throat geometries, providing an accurate representation of the turbulent flow fields. This has allowed us to examine the effect of geometric variation on the mean flow characteristics as well as the turbulence intensity, both of which affect deposition, and to relate the flow fields to in vitro deposition patterns in the same models, carried out by Grgic et al. (2004b).

A number of in vivo (Walsh et al., 1977; Foord et al., 1978; Chan \& Lippmann, 1980; Stahlhofen et al., 1980, 1981; Emmett \& Aitken, 1982; Svartengren et al., 1994; Dunbar et al., 2002) and in vitro studies (Cheng et al., 1999, 2001; Lin et al., 2001; Grgic et al., 2004b; DeHaan \& Finlay, 2004; Grgic et al., 2004a; Heenan et al., 2004; Zhang et al., 2007; Zhou et al., 2011; Shinneeb \& Pollard, 2012) have been conducted in order to develop an understanding of the flow and particle dynamics in the extrathoracic airways. In vivo experiments are costly and complex to perform, and accurate results are difficult to obtain due to spatial resolution and tissue attenuation limit (Grgic et al., 2004b). In addition, flow visualization can not be performed. In vitro measurements are relatively easier. However, most of these studies have focused solely on deposition. Grgic et al. (2004a) and Heenan et al. (2003; 2004) conducted particle image velocimetry (PIV) measurements in order to visualize the flow. This allowed comparison of the deposition patterns with the flow field, showing a strong correlation be- 
tween deposition levels and local velocity magnitude and flow curvature. However, obtaining PIV measurements in small, closed, complex geometries presents many difficulties. In addition, PIV is inherently noisy and suffers from limited resolution, so it can only capture the larger scale turbulent fluctuations. The measurements performed were also limited to the central sagittal plane due to contamination from the out-of-plane velocity component. More recently Shinneeb \& Pollard (2012) carried out PIV measurements in coronal planes across the pharynx and larynx as well, in order to gain an understanding on the characteristics of the turbulent flow. Their results showed that the flow is strongly three-dimensional, and that a large number of vortical structures occur in the pharynx/larynx region, which are deformed and torn apart by bursting events. However, stereo PIV commonly used in the literature can only provide 2D data. The recently-developed tomographic PIV technique can obtain 3D measurements but has not to date been applied to studies of the flow in the extrathoracic airways. Numerical simulations provide an alternative to PIV and can yield a more accurate and a more detailed representation of the flow.

In the last decade, computational fluid dynamics (CFD) of the flow and the particle trajectories in the extrathoracic airways has become possible, and offers a non-invasive and cost-effective alternative to in vivo and in vitro testing. A number of Reynolds-averaged Navier-Stokes (RANS) (Zhang et al., 2002; Heenan et al., 2003; Kleinstreuer \& Zhang, 2003; Matida et al., 2004; Jayaraju et al., 2007; Sandeau et al., 2010) and large eddy simulation (LES) studies (Matida et al., 2006; Jayaraju et al., 2008; Debhi, 2011; Cui \& Gutheil, 2011) have been reported in the literature. However, accurate prediction of the flow field remains a challenge due to the complexity of the flow in the extrathoracic airways and the limitations of RANS and LES turbulence models. More recently, the first direct numerical simulations in an idealized mouth-throat geometry were reported by Ball et al. (2008). The authors used a lattice Boltzmann method and demonstrated closer agreement with experimental results than RANS models.

Studies which have focused on accurately modelling the geometry have employed RANS turbulence models (Jayaraju et al., 2007; Sandeau et al., 2010) which do not resolve any of the scales in the flow, but rather model the fluctuations, usually based on empirical data 
obtained from canonical and often equilibrium flows. The flow in the complex extrathoracic airways differs significantly from a canonical duct flow however, and the inaccurate modelling of the fluctuations, which cause dispersion, affects the prediction of deposition. The effect is more significant for the smaller particles whose trajectories are considerably influenced by the fluctuations in the flow. On the other hand, LES which resolves the large scales in the flow and can therefore better predict turbulent dispersion, has been performed on simplified representations of the models (Matida et al., 2006; Jayaraju et al., 2008; Debhi, 2011; Cui \& Gutheil, 2011). Whereas Jayaraju et al. (2008) found considerable improvement in predicting deposition for smaller particles and Cui \& Gutheil (2011) showed better predictions particularly in the transitional regime using LES over RANS, Debhi (2011) obtained comparable accuracy between RANS and LES models. In addition, experiments have demonstrated the large effect that geometric complexity has on the flow, and hence deposition (Heenan et al., 2004; Grgic et al., 2004b). Therefore the simplified geometries adopted in earlier work is not sufficient. To date, there has not been a complete study including both a realistic geometry and a realistic representation of the turbulent field.

The importance of understanding and accurately capturing the flow dynamics in the extrathoracic airways is further supported by the dependence of deposition on Reynolds number, Re. This dependence was first observed by Grgic et al. (2004a) in in vitro experiments in an idealized geometry. Previously, deposition had been reported in terms of its dependence on Stokes number, Stk, alone. However, keeping the Stokes number constant and varying the Reynolds number, Re, Grgic et al. (2004a) observed an increase in deposition with Re. Based on PIV measurements, they were able to gain insight into this dependence and attributed it to the difference in velocity profile at different flow rates. Deposition efficiency was shown to depend on $S t k R e^{n}$. This scaling leads to better collapse of extrathoracic deposition onto a single curve, as opposed to the Stokes number alone. However, the physical significance of this Reynolds number correction was not explained. Examination of the flow fields in a number of realistic mouth-throat geometries allows us to address the origin of this Reynolds number dependence.

The present work is the first set of direct numerical simulations of the flow in realistic 
extrathoracic airways. Through realistic inflow conditions, geometric representation of the airways, and accurate solution of the flow field which is fully resolved, this study allows us to evaluate the effect of geometric variation on the mean flow as well as the turbulent fluctuations. Relating the flow fields to in vitro deposition data from the literature provides insight as to how geometric variation affects aerosol deposition, and helps to explain the scatter in deposition data reported in the literature. Finally, we explain the physical significance of the Reynolds number effect on deposition.

The paper is organized as follows: The geometries and the flow parameters used in this study are presented in section 2. In section 3, the numerical method is described in detail. In Section 4, the flow fields in the various mouth-throat geometries are presented and the effect of intrasubject and intersubject variation on the mean flow and the turbulence intensity is examined. The flow fields are used to explain the in vitro deposition data of Grgic et al. (2004b), obtained in the same geometries. The derivation of the Reynolds number dependence of deposition is given next. Finally, section 5 is a summary of the work.

\section{Mouth-throat geometries and flow parameters}

A set of four physiologically realistic mouth and throat geometries were used in this study. It is a subset of the geometries used by Grgic et al. (2004b) for their in vitro measurements. The anatomically accurate models were obtained using magnetic resonance imaging (MRI). The methodology is explained in detail by McRobbie et al. (2003). The MRI scans were converted to 3D volume files from which STL models were generated. The geometries can be divided into four sub-regions (see figure 1):

(1) the mouth region from the back of the teeth to the soft palate;

(2) the nasopharynx region incorporating the nasal airways to the tip of the epiglottis;

(3) the larynx, from the tip of the epiglottis to just below the vocal cords;

(4) the trachea to a point two vertebrae below the vocal cords.

The geometries studied here are denoted S1b, S1a, S2 and S4, in keeping with the labels used by Grgic et al. (2004b). They are shown in figure 2. Cases S1b and S1a represent the 
same subject and correspond to different configurations: In S1b, the tongue is in the forward position touching the back of the teeth, whereas in S1a, the tongue is pulled back creating a large mouth opening and reducing the size of the nasopharynx. Models S2 and S4 belong to different individuals, both with large oral cavities. In S2, the mouth narrows at the back and the pharynx is wider. Subject S4 has a wider mouth and a narrow pharynx with a bending angle close to $90^{\circ}$. The choice of geometries allows us to investigate the effect of both intrasubject and intersubject variation on the flow, and to explain the dependence of particle deposition on Reynolds number, as reported in the literature.

The main dimensions of the models are listed in table 1 . In order to calculate the sagittal length, $L$, the geometries were split along the central sagittal plane. Because the geometries are not symmetrical, the mid-plane was chosen such that each of the two halves of the geometry contained approximately half the volume of the model. Once cut, the path line along the centre of the geometry was measured on that plane in order to determine the length. Assuming a circular mean cross-sectional area, an equivalent mean diameter was computed for each model according to,

$$
D_{\text {mean }}=2 \sqrt{\frac{V}{\pi L}}
$$

where $V$ is the volume of the geometry.

Direct numerical simulations in the four geometries were carried out at a flow rate $Q=$ $30 \mathrm{~L} / \mathrm{min}$. The main flow parameters in the different models are summarized in table 2. The mean velocity is determined from the volume flow rate and the mean cross-sectional area according to,

$$
U_{\text {mean }}=\frac{Q L}{V},
$$

and the Reynolds number based on the mean diameter and the mean velocity in the mouththroat geometry is given by

$$
R e_{\text {mean }}=\frac{D_{\text {mean }} U_{\text {mean }}}{\nu},
$$

where $\nu$ is the kinematic viscosity of the fluid. 


\section{Numerical method}

Due to the complexity of the mouth-throat geometries, an immersed boundary (IB) method is employed. IB methods employ structured, non-conforming grids and incorporate the boundary conditions by modifying the Navier-Stokes equations near the wall of the geometry. This approach greatly simplifies the task of grid generation and discretization of the governing equations, and eliminates the problems associated with grid quality that exist with boundary-fitted grid techniques. The IB approach also allows the use of a structured grid solver, which is advantageous from the point of view of computational efficiency and scalability on high performance computing facilities, in comparison to unstructured grid methods.

In order to accurately resolve the flow, a high grid resolution is required inside the geometries. Due to the shape of the extrathoracic airways, the use of Cartesian grids which are commonly adopted in IB methods leads to many unused grid points outside the flow domain (see figure $3 a$ ). Even when grid stretching is applied, it is difficult to cluster the points efficiently inside the geometry. For turbulent flow, which is the case at the flow rate considered herein, the resolution requirements become prohibitively expensive. A more efficient alternative is therefore to adopt a curvilinear grid that roughly follows the shape of the geometry. This greatly reduces the number of points outside the geometry, thus allowing for much higher resolution within the geometries than the Cartesian grid case (see figure $3 b$ ). An added advantage of the curvilinear mesh is that the grid lines are approximately aligned with streamlines, which is favourable from a numerical accuracy point of view as this tends to reduce numerical diffusive errors.

Initial computations were performed on a grid with $14 \times 10^{6}$ cells $(385 \times 193 \times 193$ grid points). Based on the results from the coarse mesh computations, the grid was refined in the streamwise $\xi$, cross-stream $\eta$ and spanwise $z$ directions. The final, fine mesh computation included $42 \times 10^{6}$ cells $(513 \times 321 \times 257$ grid points $)$, with uniform grid spacing in the spanwise direction, $\Delta z=0.0094$, and hyperbolic tangent stretching in the streamwise and wall-normal directions in order to minimize the number of points outside the geometry 
and provide adequate resolution near the walls, $0.01<\Delta \xi<0.06$ (near the outflow) and $0.02<\Delta \eta<0.008$. Using the friction velocity at the inlet pipe, this corresponds to $\Delta z^{+}=3.12,3.32<\Delta \xi^{+}<19.9$ (near the outflow) and $0.66<\Delta \eta^{+}<2.65$ in wall units, which falls in the range typically used in the literature for turbulent internal flows. The time step is $\Delta t^{+}=0.024$, which is significantly smaller than the Kolmogorov time scale and hence adequate for time-resolved computations. Choi \& Moin (1994) demonstrated accurate prediction of turbulence statistics for $\Delta t^{+}<0.4$. The overall run-time for a simulation on 128 processors was approximately 300 hours, including the initial time to remove the transient and the time to compute statistics.

The no-slip condition at the immersed boundary is applied via a direct forcing approach similar to that by Kim et al. (2001), which consists in adding a momentum forcing term, $\boldsymbol{f}$, on the boundary and inside the solid domain. The forcing ensures that the velocity at the surface of the immersed body satisfies the boundary conditions. A mass source/sink, $q$, is applied to cells containing the immersed boundary in order to ensure mass conservation. The governing equations in non-dimensional form are therefore given by

$$
\begin{aligned}
\frac{\partial \boldsymbol{u}}{\partial t}+(\boldsymbol{u} \cdot \nabla) \boldsymbol{u} & =-\nabla p+\frac{1}{R e_{\text {mean }}} \nabla^{2} \boldsymbol{u}+\boldsymbol{f} \\
\nabla \cdot \boldsymbol{u}-q & =0
\end{aligned}
$$

where $\boldsymbol{u}=(u, v, w)$ is the velocity vector, $p$ is the pressure, $\boldsymbol{f}$ is the momentum forcing vector and $q$ is the mass source/sink.

The equations are solved on a staggered curvilinear grid using a finite volume scheme, following the method described in Rosenfeld et al. (1991). Time integration is performed with a second-order fractional step method: First, a provisional velocity $\hat{\boldsymbol{u}}$ is computed and then a pseudo-pressure, $\phi$, is used to correct the provisional velocity field so that the continuity equation is satisfied at each computational time step (Kim \& Moin, 1985).

The diffusive terms are treated implicitly using the Crank-Nicholson scheme, in order to avoid the restrictive viscous stability condition, $\Delta t \leq \frac{\Delta x^{2}}{\nu}$. The non-linear convective terms are treated explicitly using an Adams-Bashforth scheme. The discretized equations 
are given by

$$
\begin{aligned}
\frac{\hat{\boldsymbol{u}}-\boldsymbol{u}^{n-1}}{\Delta t}=-\left(\gamma N\left(\boldsymbol{u}^{n-1}\right)+\delta N\left(\boldsymbol{u}^{n-2}\right)\right) & -\nabla p^{n-1}+\frac{1}{R e_{\text {mean }}}\left(\alpha L(\hat{\boldsymbol{u}})+\beta L\left(\boldsymbol{u}^{n-1}\right)\right)+\boldsymbol{f}^{n} \\
\nabla^{2} \phi^{n} & =\frac{1}{\Delta t}\left(\nabla \cdot \hat{\boldsymbol{u}}-q^{n}\right) \\
\boldsymbol{u}^{n} & =\hat{\boldsymbol{u}}-\Delta t \nabla \phi^{n} \\
p^{n} & =p^{n-1}+\phi^{n}
\end{aligned}
$$

where $N(\boldsymbol{u})$ are the convective terms, $L(\boldsymbol{u})$ are the implicit diffusive terms and $(\alpha, \beta, \gamma, \delta)$ are weighting coefficients which depend on the numerical scheme adopted. In our case, $\alpha=3 / 2$, $\beta=-1 / 2$ for the Adams-Bashforth scheme and $\gamma=\delta=1 / 2$ for the Crank-Nicholson scheme.

The inflow condition: The flow at the inlet to the computational domain was designed based on the Reynolds number, in order to match the experimental flow setup. For geometries $\mathrm{S} 2$ and S4, since the Reynolds number is in the laminar regime, a parabolic velocity profile is prescribed at the inlet. For S1a and S1b, where the flow in the inlet tube is turbulent, accurate turbulent inflow conditions are required. These were obtained from a separate direct numerical simulation of pipe flow at the Reynolds number reported in the experiments.

The length of the pipe is $L_{x}=5 D_{\text {inlet }}$, which is sufficiently long to include the largest structures in the flow (Eggels et al., 1994). A $256 \times 128 \times 64$ grid is employed, with uniform spacing in the streamwise and circumferential directions, $\Delta x^{+}=4.55,(r \Delta \theta)^{+}=5.72$, and stretching in the radial direction in order to provide adequate resolution near the walls, $0.09 \leq \Delta r^{+} \leq 4.96$. Similar grid resolutions have previously been adopted in the literature (Kim et al., 1987; Eggels et al., 1994). Starting from a laminar flow field with superimposed initial fluctuations, fully-developed turbulence is obtained after ten flow-through times.

By invoking Taylor's hypothesis (Taylor, 1938), convective spatial fluctuations can be interpreted as temporal fluctuations. In this manner, the axial pipe coordinate can be transformed into a time coordinate. The first cross-sectional plane from the turbulent pipe flow is applied as an inflow condition to the mouth at the initial time, and the time-dependence 
of the inflow is emulated by sweeping through planes along the pipe axis. The mean velocity profile and the mean turbulence intensities at the inlet are shown in figure 4 .

The outflow condition: At the outflow boundary, a convective condition is applied. This is given by

$$
\frac{\partial \boldsymbol{u}}{\partial t}=-c \frac{\partial \boldsymbol{u}}{\partial n} ; \quad c<0
$$

where $c$ is the convective velocity, chosen to be the bulk velocity at the outlet, and $n$ is the direction normal to the exit plane. An extension approximately two mean diameters long was added to the outlet of the mouth-throat geometries for two reasons: Firstly, the extended domain ensures that any inaccuracies in estimating the outflow conditions are not propagated upstream into the region of interest. Secondly, the extension ensures that the outflow plane is downstream of any separation zone.

The instantaneous velocity field $\boldsymbol{u}$ comprises a mean component $\overline{\boldsymbol{u}}$ and a fluctuating component $\boldsymbol{u}^{\prime}$ :

$$
\boldsymbol{u}=\overline{\boldsymbol{u}}+\boldsymbol{u}^{\prime}
$$

Snapshots of the instantaneous velocity magnitude and the magnitude of fluctuations in one of the geometries are shown in figure 5. It illustrates the turbulent nature of the flow. The mean velocity is responsible for the convective transport of aerosol particles, whereas the fluctuations are responsible for turbulent dispersion. Understanding the deposition of particles in the extrathoracic airways therefore requires inspection of both the mean and the fluctuating components of the velocity.

The mean velocities and root-mean-square of the fluctuating components are computed with a running average in time:

$$
\begin{gathered}
\overline{\boldsymbol{u}}=\frac{1}{T} \int_{0}^{T} \boldsymbol{u} d t \\
\boldsymbol{u}_{\mathrm{rms}}=\left(\overline{\boldsymbol{u}^{\prime 2}}\right)^{\frac{1}{2}}=\left(\frac{1}{T} \int_{0}^{T} \boldsymbol{u}^{2} d t-\overline{\boldsymbol{u}}^{2}\right)^{\frac{1}{2}}
\end{gathered}
$$

where $T$ is the time period for statistical convergence. We study the effects of intrasubject and intersubject variation on the flow field by comparison of the mean flow patterns and the mean turbulent kinetic energy per unit mass, $k=\frac{1}{2}\left(\overline{u^{\prime 2}}+\overline{v^{\prime 2}}+\overline{w^{\prime 2}}\right)$, across geometries. 


\section{Results and discussion}

\subsection{Intrasubject variation}

Comparison between geometries S1b and S1a shows how intrasubject variation can account for significant differences in the flow field. Figures $6 a$ and $7 a$ show contours of the mean velocity magnitude in the central sagittal plane and at various cross-sections in models S1a and S1b respectively. Two-dimensional streamlines in the corresponding planes are plotted in figures $6 b$ and $7 b$ respectively.

In S1b, the velocity profiles in the mouth are highly skewed towards the inner wall due to the airway curvature (A1-A2). The flow accelerates at the back of the mouth due to the restriction in cross-sectional area, developing a pharyngeal jet which impinges onto the posterior wall (B1-B2). Due to the bend in the airway, the flow shifts towards the outer wall, separating from the inner wall and leading to a recirculation region. The maximum velocity then decreases as the larynx expands in the spanwise direction, and flow moves into the lateral expansions (C1-C2). A small recirculation region at the posterior side of the exit to the trachea can be observed under the 'sharp step' in the larynx (upstream of E1-E2).

Due to the wider oral cavity in model S1a, there is no pharyngeal jet (B1-B2). As a result, the velocity in the pharynx is lower and the flow does not separate from the inner wall. Instead, the flow accelerates further downstream resulting in higher velocities in the larynx (D1-D2), due to the narrower nasopharynx than in S1b. This in turn leads to a larger separation region near the outer wall of the trachea (E1-E2).

Heenan et al. (2004) conducted PIV measurements of the flow in the same geometries at both $Q=30$ and $90 \mathrm{~L} / \mathrm{min}$. They asserted that the flow was qualitatively similar for the two flow rates, and discussed the case with $Q=90 \mathrm{~L} / \mathrm{min}$ in detail. Therefore, their results can be used for qualitative comparison of the main flow features. The PIV measurements in both geometries display the same mean flow characteristics as the flow fields presented herein. In S1b, both DNS and PIV results show the existence of a pharyngeal jet impinging on the posterior wall in the upper pharynx (figure 6 herein and figure 4 by Heenan et al. (2004)). In S1a, we note the absence of a jet in both experimental and numerical results, 
and an increase in the velocity further downstream in the larynx (figure 7 herein and figure 6 by Heenan et al. (2004)). The only discrepancy between the two sets of results is in the oral cavity in S1a, where the impingement of the inlet flow on the front of the tongue is inappreciable in the PIV measurements due to the wider inlet pipe used in the experiments. However, as can be seen from the results and has also previously been reported in the literature, the inflow shows no appreciable effect on the flow downstream (Ball et al., 2008). Finally, the separated shear layer in the trachea of S1a was not reported by Heenan et al. (2004) as their PIV measurements focused on the upstream region of the flow.

In addition to the agreement with the PIV data in the central sagittal plane, the current computations have provided a high-resolution representation of the flow field throughout the extrathoracic airways, for example in the coronal cross-sections discussed above. The direct numerical simulations also provide accurate characterization of the turbulence. Contours of the turbulent kinetic energy per unit mass in S1b and S1a are shown in figure $8 a$ and $b$ respectively. The plots show that the turbulence intensity is higher in model S1a, even though the Reynolds number is lower. This is due to the inflow condition and the geometry of the airways. The flow in the mouth in S1b is similar to pipe flow, whereas in S1a a jet from the inlet pipe impinges against the tongue. Separation occurs at the upper wall due to the wide oral cavity, and under the jet where the flow resembles that over a backwardfacing step. Higher levels of turbulent kinetic energy can be observed near the regions of separation. The maximum kinetic energy in S1b occurs in the upper pharynx near the jet, and in S1a, in the larynx. High values of $k$ can also be observed downstream of the vocal cords (see the sagittal plane in figure $8 a$ ). These are locations where separated shear layers exist. Generally, turbulence is strongest where mean shear is largest.

The flow fields can be used to explain the in vitro deposition results of Grgic et al. (2004a). The deposition fractions in the different regions of geometries S1a and S1b are given in table 3 . For all three particle sizes the main difference between the two geometries is the increased pharyngeal deposition in S1b. This is due to the pharyngeal jet, which causes particles to deposit on the posterior wall of the upper pharynx via impaction.

Deposition levels for the smallest $(3 \mu \mathrm{m})$ and the largest $(6.5 \mu \mathrm{m})$ particles are very similar 
in both geometries. For the smallest particles, slightly higher deposition in the mouth and pharynx is observed in S1b. Not only do particles deposit via impaction at the back of the mouth and the upper pharynx, but in addition, the high levels of turbulence intensity on either side of the jet (see figure 8b) cause particle dispersion, causing small particles travelling near the wall in the upper pharynx to deposit. In S1a, high turbulent kinetic energy can be observed in the larynx, which explains the slightly higher deposition (via dispersion) in this region, compared to S1b. It is clear that both the mean flow characteristics and the velocity fluctuations play a role in the deposition of the small particles.

Large particles are less influenced by the velocity fluctuations and therefore the main deposition mechanism is impaction. Oral deposition in S1a occurs at the front of the tongue where the incoming flow from the inlet pipe impinges, whereas in S1b it occurs mainly at the back of the mouth due to the high velocities and the airway curvature. For the medium-sized particles, deposition in the mouth, pharynx and larynx is significantly higher in S1b, via impaction due to the pharyngeal jet. The fact that this significant difference in deposition between S1a and S1b isn't also observed for the largest-sized particles is somewhat anomalous, as mentioned by Grgic et al. (2004a), who cited the large variation in regional deposition and flow field measurements reported by Heenan et al. (2004). Deposition in the trachea is higher in S1a due to the high-velocity separated shear layer that forms at the sharp step in the larynx (see location D1-D2 in figure 6).

\subsection{Intersubject variation}

Geometries S2 and S4 have the same inlet diameter and the same mean Reynolds number, making the comparison between the two geometries a good test of the effect of geometric variation on the flow field. Both geometries are considerably different to S1a and S1b. This results in significant differences in the flow fields, which can be observed in the velocity contours shown in figures $9 a$ and $10 a$, and in the streamlines plotted in figures $9 b$ and $10 b$. The velocities reach much higher levels when normalized by the reference speed, as the variation in the cross-sectional area is much larger. However, absolute values of velocity are lower as the geometries on average are wider (see table 1). 
In $\mathrm{S} 2$, the velocities in the mouth are low due to the wide oral cavity, with recirculation at the top and bottom walls near the inlet. Strong secondary flow exists, as the fluid coming in from the pipe moves radially outwards to fill the wide area (A1-A2). The fluid then accelerates at the back of the mouth and upper pharynx due to the large reduction in cross-sectional area (B1-B2). An obstruction in the centre of the airway causes the flow to split into two diverging jets (C1-C2), shifting the flow towards the side walls (D1-D2). Four distinct counter-rotating vortices can be observed at this location: two main cells at the centre, and two smaller cells near the side walls. The main cells resemble Dean vortices known to appear in curved ducts as a result of centrifugal instability. Smaller secondary cells have also been observed in curved pipe flow studies (Daskopoulos \& Lenhoff, 1989). Further downstream, the secondary flow weakens and the velocity drops due to an increase in crosssectional area (E1-E2). A small recirculation region develops near the anterior wall due to the sharp expansion. The flow in the trachea is similar to S1a, with the fluid accelerating again as it passes the sharp bend (F1-F2) and a small separation region developing near the posterior wall (G1-G2).

Similar to model S2, the velocity in the mouth of geometry S4 is low and strong secondary flow exists, due to the large oral cavity (A1-A2). The back of the mouth is wider than in S2, so velocities there are lower (B1-B2). Instead, the flow accelerates further downstream, after the sharp $90^{\circ}$ bend into the pharynx (C1-C2). The velocity remains roughly constant throughout the pharynx as the cross-sectional area does not change appreciably. The small expansion near the anterior wall is sudden so the flow remains unaffected and attached to the posterior wall (D1-D2). A slight drop in the velocities is observed in the larynx, where the cross-sectional area is larger (E1-E2), before an increase in the trachea as the airway narrows and bends (F1-F2). The trachea is shorter and narrower than in the other geometries, therefore velocities are still high at the exit (G1-G2).

Although the inflow is laminar based on the inlet Reynolds number, the flow in both geometries transitions to turbulence at the back of the mouth, as can be seen in figure $11 a$ and $b$. The contours of turbulent kinetic energy in S2 show much higher levels of turbulence intensity compared to all other geometries, even though the Reynolds numbers in S1a and 
S1b are higher. The location of maximum $k$ occurs close to the core of one of the main vortices, in the upper pharynx. This is due to the strong gradients in tangential velocity inside the vortex. In $\mathrm{S} 4$, most of the turbulent kinetic energy is produced in the pharynx and larynx due to the separated shear layers. The maximum value of $k$ occurs in the upper pharynx, between the separation region and the pharyngeal jet, where shear is highest.

The flow fields can be used to explain the in vitro deposition results of Grgic et al. (2004a). The in vitro deposition fractions in the different regions of geometries S2 and S4 are given in table 4. Deposition is low in comparison with S1a and S1b, due to the lower flow inertia. We can see that the regional and total depositions differ between S2 and S4, despite the same Reynolds number in both geometries. For all three particle sizes, slightly higher total deposition is observed in S4. This is mainly due to the higher deposition in the pharynx and larynx, as the higher velocities and the bigger radius of curvature cause the particles to deposit via impaction. The difference is most significant for the largest particles, as they have more inertia, which causes more appreciable deviation from the streamlines. Although strong secondary flow exists in the mouth as the air coming in from the inlet pipe moves radially outwards, oral deposition in both geometries is low due to the low inflow velocity and the wide oral cavity. No deposition is observed for the smallest particles in geometry S2, despite the high levels of turbulent kinetic energy, which suggests that impaction is the main deposition mechanism for the range of particle sizes considered.

The flow results presented herein and the above discussion demonstrate the following:

(i) The flow in the extrathoracic airways is complex. Highly asymmetric velocity profiles, complex secondary flow and regions of separation are observed in all geometries.

(ii) Transition to turbulence occurs even if the inflow is laminar, due to the complex geometry of the airways. This transition occurs rapidly, with the flow becoming turbulent around the back of the mouth. Therefore, the effect of the inflow on the flow and the deposition in the airways is mainly confined to the oral cavity. Conventional RANS approaches are known to perform poorly in transitional flows (Stapleton et al., 2000), and careful selection of a turbulence model is required for accurate prediction of flow 
in the airways (Longest \& Vinchurkar, 2007). DNS naturally captures transition, circumventing the need to select a particular turbulence model.

(iii) Geometric variation has a large effect on the flow field. This variation affects the flow, and in turn the particle deposition, in two ways:

(a) The different shape of the airways leads to different flow patterns and hence different "hot-spots" for particle deposition. For example, even in the case of geometries S2 and S4 in which the Reynolds numbers are the same, the mean flow patterns and the levels of turbulence intensity differ. This is because the Reynolds number is based on the mean characteristic length of the geometries, but the diameters of the geometries deviate considerably from the mean in many sections of the airways.

(b) For a given volume flow rate, the Reynolds number is different in various geometries. This changes the mean velocity profiles and the turbulence intensities which in turn affect particle trajectories and their deposition. This Reynolds number effect is the focus of the next subsection.

\subsection{Reynolds number effect}

Deposition efficiency in the extrathoracic airways is commonly examined in terms of its dependence on the Stokes number, given by

$$
S t k=\frac{\rho_{p} d_{p}^{2} U_{\text {mean }}}{18 \mu D_{\text {mean }}}
$$

where $\rho_{p}$ is the particle density, $d_{p}$ is the particle diameter and $\mu$ is the dynamic viscosity of the fluid. However, experiments carried out by Grgic et al. (2004a) in an idealized geometry demonstrated that there is also a dependence of deposition on the Reynolds number. They observed that the deposition efficiency at two different flow rates lay on different curves when plotted against Stokes number, indicating a possible Reynolds number effect on deposition. For this reason, another set of experiments was carried out where Stokes number was held constant and Reynolds number was varied. Deposition was seen to increase with increased Reynolds number. An empirical Reynolds number correction $R e^{0.37}$ that collapsed deposition data more closely onto a single curve was proposed. Using this correlation, a number of in 
vitro experiments have been plotted against $S t k R e^{0.37}$ in the literature (see figure 11 in Grgic et al., 2004a), and showed good collapse. The Reynolds number dependence in the work of Grgic et al. (2004a) was motivated by empirical observations and data-fitting, but its physical significance was not explained. A theoretical, or fluid-dynamical, explanation is presented herein.

As mentioned in the previous subsection, the Reynolds number dependence is due to at least two contributing factors: (1) the difference in the mean flow characteristics and, (2) the difference in turbulence intensity. This was observed in the difference in mean flow features and turbulent kinetic energy among the different geometries. Whereas the effect of the mean flow field is already taken into account in the Stokes number, the level of turbulence intensities is not. Therefore, the Stokes number only accounts for impaction as a deposition mechanism, and does not include the dispersion experienced by the particles due to turbulence fluctuations. Deposition should be plotted against a parameter that takes both deposition mechanisms into account. Plotting against the Stokes number, which only account for impaction, contributes a reason for the scatter in deposition data which has been observed in the literature.

For canonical turbulent flows, such as channel and pipe flow, particle deposition is well documented in the literature (Liu \& Agarwal, 1974; Kallio \& Reeks, 1989; McLaughlin, 1989; Wang \& Squires, 1996; Young \& Leeming, 1997) and is generally plotted against the dimensionless particle relaxation time, $\tau_{p}^{+}$, given by

$$
\tau_{p}^{+}=\frac{\tau_{p} u_{\tau}^{2}}{\nu}
$$

Here, $\tau_{p}=\frac{\rho_{p} d_{p}^{2}}{18 \mu}$ is the particle relaxation time and $u_{\tau}=\left(\frac{\tau_{w}}{\rho}\right)^{\frac{1}{2}}$ is the friction velocity, where $\tau_{w}$ is the wall shear stress and $\rho$ is the fluid density.

The dimensionless particle relaxation time takes into account both impaction and turbulent diffusion, and is herein used to prove the dependence of deposition on Reynolds number. Equation 15 can be rewritten as,

$$
\tau_{p}^{+}=S t k \frac{D_{\text {mean }}}{U_{\text {mean }}} \frac{u_{\tau}^{2}}{\nu}
$$


where $\frac{D_{\text {mean }}}{U_{\text {mean }}}$ is the mean flow time scale and $\frac{\nu}{u_{\tau}^{2}}$ is the viscous time scale. Since fluctuations scale roughly with the friction velocity $u_{\tau}$, the viscous time scale can be seen as representing the fluctuation time scale. Next, an expression for the friction velocity $u_{\tau}$ is sought in terms of $R e$.

For fully-developed turbulent pipe flow, a relation between the friction velocity and the mean velocity can be obtained through the expression for the friction factor,

$$
f \equiv \frac{4 \tau_{w}}{\frac{1}{2} \rho U_{\text {mean }}^{2}}=8\left(\frac{u_{\tau}}{U_{\text {mean }}}\right)^{2} .
$$

Blasius (1913) derived a fit for the experimental data, given by

$$
f=\frac{0.3164}{R e^{n}}
$$

where $n=0.25$, from which an expression for the friction velocity can be obtained

$$
u_{\tau}^{2}=\frac{0.3164 U_{\text {mean }}^{2}}{8 R e^{n}}
$$

Substituting into equation 16 yields

$$
\tau_{p}^{+}=\frac{0.3164}{8} S t k R e^{1-n}
$$

Equation 20 gives the non-dimensional relaxation time in terms of Stokes and Reynolds numbers, showing the dependence of deposition on these two parameters. Unfortunately, this is only valid for fully-developed, smooth, turbulent pipe flow. A similar expression for the mouth and throat cannot be derived analytically as there is no such simple relation for $u_{\tau}$, partly because it is inhomogeneous due to the complexity of the geometry and partly because of the transitional nature of the flow. However, it explains the Reynolds number dependence of deposition due to the turbulent nature of the flow. Other flow characteristics, such as the extent of separated regions and jettal flow structures could also be contributing to the Reynolds number effect on deposition. This affirms the importance of an accurate representation of the flow field and the value in performing DNS which captures all the scales in the flow.

The empirical correlation of Grgic et al. (2004b), StkRe $e^{0.37}$, suggests that the ratio of friction velocity to mean velocity decays faster with Reynolds number in the mouth and 
throat, $\left(u_{\tau} / U\right)^{2} \propto R e^{-0.63}$, than it does in a pipe, $\left(u_{\tau} / U\right)^{2} \propto R e^{-0.25}$. The derivation of the latter has herein assumed an expression for the friction factor (equation 18) which applies only in fully-turbulent pipe flow conditions. It is therefore expected to differ from the flow in the extrathoracic airways which is spatially-developing, transitional, and includes features such as separation and impinging jets.

\section{Summary}

The work presented herein is the first set of direct numerical simulations of the flow in realistic extrathoracic airways, and the first numerical study of the effect of geometric variation on the flow. The results demonstrate that the flow in the extrathoracic airways is complex due to their geometry, which varies significantly across subjects. For a given flow rate, this geometric variation leads to different Reynolds numbers, which results in different flows and leads in turn to different deposition patterns. Variation is observed even within the same subject, where the position of the tongue can create significant geometric differences resulting in qualitatively different flow features. For example, the impinging pharyngeal jet present in geometry S1b does not exist in S1a, where the tongue is pulled back. This variation is even more pronounced across subjects, as is observed by comparison of geometries S2 and S4 with S1a and S1b. Differences in the mean flow (impinging jets, separated shear layers, vortical patterns) as well as in the velocity fluctuations can be observed, both of which have an effect on particle trajectories and deposition.

The dependence of deposition on the Reynolds number, observed in the in vitro experiments conducted by Grgic et al. (2004b), is due to the difference in the mean flow field as well as the difference in the turbulence intensities. The better collapse of deposition data observed in the literature, when plotted against the empirical correlation $S t k R e^{0.37}$ rather than Stk alone, is due to the fact that the Stokes number fails to account for the level of turbulence intensity in the airways. Therefore, it only takes into account impaction as a deposition mechanism, and does not include the turbulent dispersion experienced by the aerosol particles due to velocity fluctuations. By use of the dimensionless particle relaxation 
time, $\tau_{p}^{+}$, which takes into account both these deposition mechanisms, the origin of this Reynolds number dependence was explained.

Although the Reynolds number correction improves the collapse of deposition data, scatter is still observed in the literature. An explanation is provided by considering intersubject variation of the flow field. Even at the same Reynolds number and flow rate, as is the case in geometries S2 and S4, flow features can differ significantly due to geometric variation. Therefore, plotting deposition against Stokes and Reynolds numbers based on mean length and velocity scales does not entirely eliminate scatter, since the diameters of the geometries, and hence the velocities, deviate considerably from the mean in many sections of the airways. The question of appropriate scaling for flow in the extrathoracic airways has been a point of discussion in the literature (Ball et al., 2008) and remains a challenge in providing an accurate model of extrathoracic deposition, which reinforces the importance of efficient subject-specific prediction.

\section{Acknowledgements}

The authors would like to thank GlaxoSmithKline (N. E. Stevens, D. Prime and M. Palmer). This study was funded by GlaxoSmithKline (UK) and the Engineering and Physical Scieces Research Council (EPSRC).

Ball, C., Uddin, M., \& Pollard, A. (2008). Mean flow structures inside the human upper airway. Flow, Turbulence and Combustion, 81, 155-188.

Blasius, H. (1913). Das Ähnlichkeitsgesetz bei Reibungsvorgängen in Flüssigkeiten. Forschungs-Arbeit des Ingenieur-Wesens, 131.

Chan, T. L., \& Lippmann, M. (1980). Experimental measurements and empirical modelling of the regional deposition of inhaled particles in humans. American Industrial Hygiene Association Journal, 41, 399-409.

Cheng, Y., Fu, C., Yazzie, D., \& Zhou, Y. (2001). Respiratory deposition patterns of salbutamol pMDI with CFC and HFA-134a formulations in a human airway replica. Journal of Aerosol Medicine, 14, 255-266.

Cheng, Y. S., Zhou, Y., \& Chen, B. T. (1999). Particle deposition in a cast of human oral airways. Aerosol Science and Technology, 31, 286-300.

Choi, H., \& Moin, P. (1994). Effects of the computational time step on numerical solutions of turbulent flow. Journal of Computational Physics, 113, 1-4. 
Cui, X. G., \& Gutheil, E. (2011). Large eddy simulation of the unsteady flow-field in an idealised human mouth-throat configuration. Journal of Biomechanics, 44, 2768-2774.

Daskopoulos, P., \& Lenhoff, A. M. (1989). Flow in curved ducts: bifurcation structure for stationary ducts. Journal of Fluid Mechanics, 203, 125-148.

Debhi, A. (2011). Prediction of extrathoracic aerosol deposition using RANS-random walk and LES approaches. Aerosol Science and Technology, 45, 555-569.

DeHaan, W., \& Finlay, W. (2004). Predicting extrathoracic deposition from dry powder inhalers. Journal of Aerosol Science, 35, 309-331.

Dunbar, C., Scheuch, G., Sommerer, K., DeLong, M., Verma, A., \& Batycky, R. (2002). In vitro and in vivo dose delivery characteristics of large porous particles for inhalation. International Journal of Pharmaceutics, 245, 179-189.

Eggels, J. G., Unger, F., Weiss, M. H., Westerweel, J., Adrian, R. J., Friedrich, R., \& Nieuwstadt, F. T. M. (1994). Fully developed turbulent pipe flow: a comparison between direct numerical simulation and experiment. Journal of Fluid Mechanics, 268, 175-209.

Emmett, P. C., \& Aitken, R. J. (1982). Measurements of the total and regional deposition of inhaled particles in the human respiratory tract. Journal of Aerosol Science, 13, 549-563.

Foord, N., Black, A., \& Walsh, M. (1978). Regional deposition of 2.5-7.5 $\mu \mathrm{m}$ diameter inhaled particles in healthy male non-smokers. Journal of Aerosol Science, 9, 343-357.

Grgic, B., Finlay, W., Burnell, P., \& Heenan, A. (2004a). In vitro intersubject and intrasubject deposition measurements in realistic mouth-throat geometries. Journal of Aerosol Science, 35, 1025-1040.

Grgic, B., Finlay, W., \& Heenan, A. (2004b). Regional aerosol deposition and flow measurements in an idealized mouth and throat. Journal of Aerosol Science, 35, 21-32.

Heenan, A., Finlay, W., Grgic, B., Pollard, A., \& Burnell, P. (2004). An investigation of the relationship between the flow field and regional deposition in realistic extra-thoracic airways. Journal of Aerosol Science, 35, 1013-1023.

Heenan, A., Matida, E., Pollard, A., \& Finlay, W. (2003). Experimental measurements and computational modeling of the flow filed in an idealized human oropharynx. Experiments in Fluids, 35, 70-84.

Jayaraju, S. T., Brouns, M., Lacor, C., Belkassem, B., \& Verbanck, S. (2008). Large eddy and detached eddy simulations of fluid flow and particle deposition in a human mouth-throat. Journal of Aerosol Science, $39,862-875$.

Jayaraju, S. T., Brouns, M., Verbanck, S., \& Lacor, C. (2007). Fluid flow and particle deposition analysis in a realistic extrathoracic airway model using unstructured grids. Journal of Aerosol Science, 38, 494-508.

Kallio, G. A., \& Reeks, M. W. (1989). A numerical simulation of particle deposition in turbulent boundary layers. International Journal of Multiphase Flow, 15, 433-446. 
Kim, J., Kim, D., \& Choi, H. (2001). An immersed-boundary finite-volume method for simulations of flows in complex geometries. Journal of Computational Physics, 171, 132-150.

Kim, J., \& Moin, P. (1985). Application of a fractional-step method to incompressible Navier-Stokes equations. Journal of Computational Physics, 59, 308-323.

Kim, J., Moin, P., \& Moser, R. (1987). Turbulence statistics in fully developed channel flow at low Reynolds number. Journal of Fluid Mechanics, 177, 133-166.

Kleinstreuer, C., \& Zhang, Z. (2003). Laminar-to-turbulent fluid-particle flows in a human airway model. International Journal of Multiphase Flow, 29, 271-289.

Lin, T. C., Breysee, P. N., Laube, B. L., \& Swift, D. L. (2001). Mouthpiece diameter affects deposition efficiency in cast models of the human oral airways. Journal of Aerosol Medicine, 14, 335-341.

Liu, B. Y. H., \& Agarwal, J. K. (1974). Experimental observation of aerosol deposition in turbulent flow. Journal of Aerosol Science, 5, 145-155.

Longest, P. W., \& Vinchurkar, S. (2007). Validating CFD predictions of respiratory aerosol deposition: Effects of upstream transition and turbulence. Journal of Biomechanics, 40, 305-316.

Matida, E., Finlay, W., Breuer, M., \& Lange, C. (2006). Improving prediction of aerosol deposition in an idealized mouth using large-eddy simulation. Journal of Aerosol Medicine, 19, 290-300.

Matida, E., Finlay, W., Lange, C., \& Grgic, B. (2004). Improved numerical simulation of aerosol deposition in an idealized mouth-throat. Journal of Aerosol Science, 35, 1-19.

McLaughlin, J. B. (1989). Aerosol particle deposition in numerically simulated channel flow. Physics of Fluids A, 1, 1211-1224.

McRobbie, D. W., Pritchard, S., \& Quest, R. A. (2003). Studies of the human oropharyngeal airspaces using magnetic resonance imaging. I. Validation of a three-dimensional MRI method for producing ex vivo virtual and physical casts of the oropharyngeal airways during inspiration. Journal of Aerosol Medicine, 16, 401-415.

Rosenfeld, M., Kwak, D., \& Vinokur, M. (1991). A fractional step solution method for the unsteady incompressible Navier-Stokes equation in generalized coordinate systems. Journal of Computational Physics, 94, 102-137.

Sandeau, J., Katz, I., Fodil, R., Louis, B., Apiou-Sbirlea, G., Caillibotte, G., \& Isabey, D. (2010). CFD simulation of particle deposition in a reconstructed human oral extrathoracic airway for air and heliumoxygen mixtures. Journal of Aerosol Science, 41, 281-294.

Shinneeb, A. M., \& Pollard, A. (2012). Investigation of the flow physics in the human pharynx/larynx regions. Experiments in Fluids, (pp. 1-15).

Stahlhofen, W., Gebhart, J., \& Heyder, J. (1980). Experimental determination of the regional deposition of aerosol particles in the human respiratory tract. American Industrial Hygiene Association Journal, 41, 
$385-398$.

Stahlhofen, W., Gebhart, J., \& Heyder, J. (1981). Biological variability of regional deposition of aerosol particles in the human respiratory tract. American Industrial Hygiene Association Journal, 42, 348-352.

Stapleton, K. W., Guentsch, E., Hoskinon, M. K., \& Finlay, W. H. (2000). On the suitability of $k-\epsilon$ turbulence modeling for aerosol deposition in the mouth and throat: A comparison with experiment. Journal of Aerosol Science, 31, 739-749.

Svartengren, K., Lindestad, P. A., Svartengren, M., Bylin, G., Philipson, K., \& Camner, P. (1994). Deposition of inhaled particles in the mouth and throat of asthmatic subjects. European Respiratory Journal, 7, 1467-1473.

Taylor, G. I. (1938). The spectrum of turbulence. Proceedings of the Royal Society of London. Series A, Mathematical and Physical Sciences, 164, 476-490.

Walsh, M., Black, A., \& Foord, N. (1977). Apparatus for the administration by inhalation of radioactive particles to human subjects for studies of deposition and clearance. Journal of Aerosol Science, 8, 83-90.

Wang, Q., \& Squires, K. D. (1996). Large eddy simulation of particle deposition in a vertical turbulent channel flow. International Journal of Multiphase Flow, 22, 667-683.

Young, J., \& Leeming, A. (1997). A theory of particle deposition in turbulent pipe flow. Journal of Fluid Mechanics, 340, 129-159.

Zhang, Y., Gilbertson, K., \& Finlay, W. H. (2007). In vivo - in vitro comparison of deposition in three mouth-throat models with Qvar and Turbuhaler inhalers. Journal of Aerosol Medicine, 20, 227-235.

Zhang, Z., Kleinstreuer, C., \& Kim, C. S. (2002). Micro-particle transport and deposition in a human airway model. Journal of Aerosol Science, 33, 1635-1652.

Zhou, Y., Sun, J., \& Cheng, Y. S. (2011). Comparison of deposition in the USP and physical mouth-throat models with solid and liquid particles. Journal of Aerosol Medicine and Pulmonary Drug Delivery, 24, 277-284. 


\section{List of Figures}

1 Sub-regions of the mouth-throat shown on the central sagittal plane. . . . . . 26

2 Geometries used in the DNS: (a) S1b, (b) S1a, (c) S2, (d) S4. . . . . . . . 27

3 Grids for model S1a. (a) Cartesian grid. Every eighth grid line in $x$ and $y$ has been plotted. (b) Curvilinear grid. Every fourth grid line in $\xi$ and eighth grid line in $\eta \ldots \ldots \ldots \ldots \ldots \ldots$. . . . . . . . . . . . . 28

4 (a) Mean streamwise velocity $\bar{u}$ normalized by the inlet bulk velocity $U_{\text {inlet }}$.

(b) Turbulence intensities normalized by the inlet bulk velocity: -,$u_{\mathrm{rms}}$; - ,$- v_{\mathrm{rms}} ;-\ldots-, w_{\mathrm{rms}} \ldots \ldots \ldots \ldots \ldots$

5 Contours of $(a)$ instantaneous velocity magnitude and $(b)$ fluctuation magnitude in geometry S1a. . . . . . . . . . . . . . . . . . . . 30

6 (a) Contours of mean velocity magnitude and (b) 2D streamlines in the central sagittal plane and at various cross-sections of geometry S1b. . . . . . . . . . 31

7 (a) Contours of mean velocity magnitude and (b) 2D streamlines in the central sagittal plane and at various cross-sections of geometry S1a. . . . . . . . . . 32

8 Contours of mean turbulent kinetic energy in the central sagittal plane and at various cross-sections of $(a)$ geometry S1b, and $(b)$ geometry S1a. . . . .

9 (a) Contours of mean velocity magnitude and (b) 2D streamlines in the central sagittal plane and at various cross-sections of geometry S2 . . . . . . . . . . 34

10 (a) Contours of mean velocity magnitude and (b) 2D streamlines in the central sagittal plane and at various cross-sections of geometry S4. . . . . . . . . .

11 Contours of mean turbulent kinetic energy in the central sagittal plane and at various cross-sections of $(a)$ geometry $\mathrm{S} 2$, and $(b)$ geometry $\mathrm{S} 4 . \ldots . . .36$ 


\section{List of Tables}

1 Dimensions of the mouth and throat geometries. . . . . . . . . . . . 37

2 Flow parameters in the mouth and throat geometries. . . . . . . . . . . 38

3 Deposition in the different regions of the S1a and S1b geometries, given as a percentage of aerosol entering the mouth (Grgic et al., 2004a). . . . . . . . . 39

4 Deposition in the different regions of the S2 and S4 geometries, given as a percentage of aerosol entering the mouth (Grgic et al., 2004a). . . . . . . . . 40 


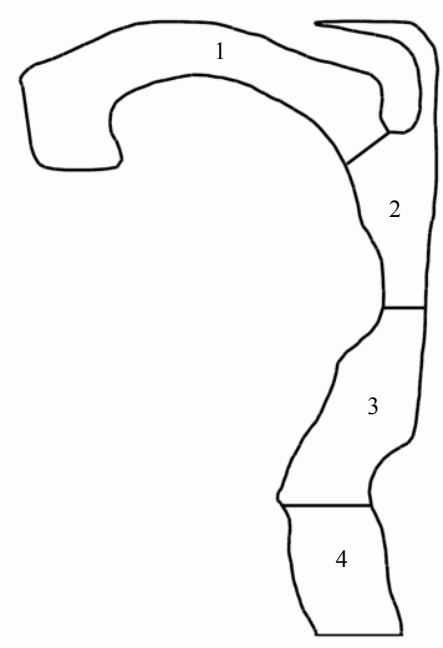

Figure 1: Sub-regions of the mouth-throat shown on the central sagittal plane. 
(a)

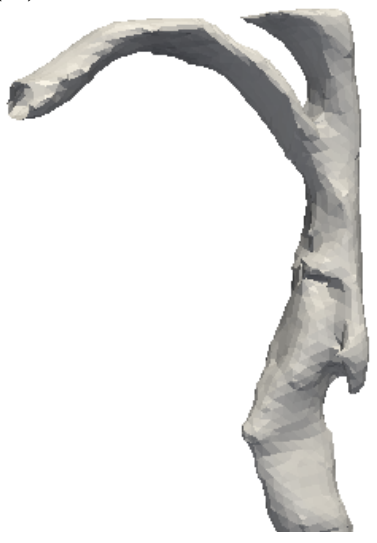

(b)

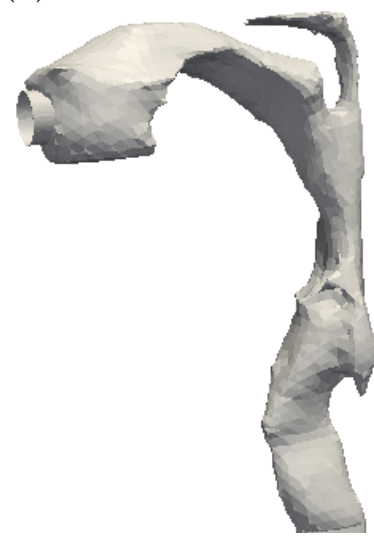

(c)

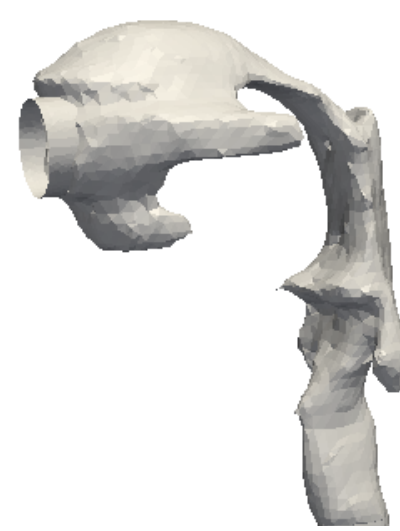

(d)

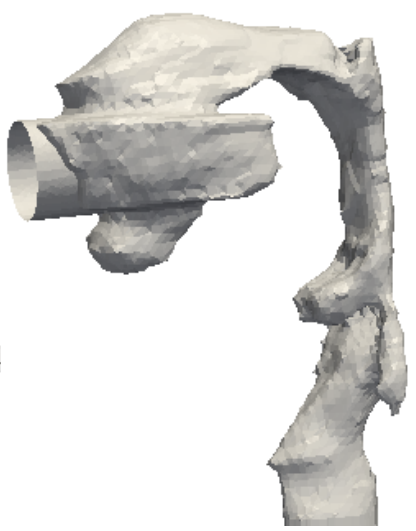

Figure 2: Geometries used in the DNS: (a) S1b, (b) S1a, (c) S2, (d) S4. 
(a)

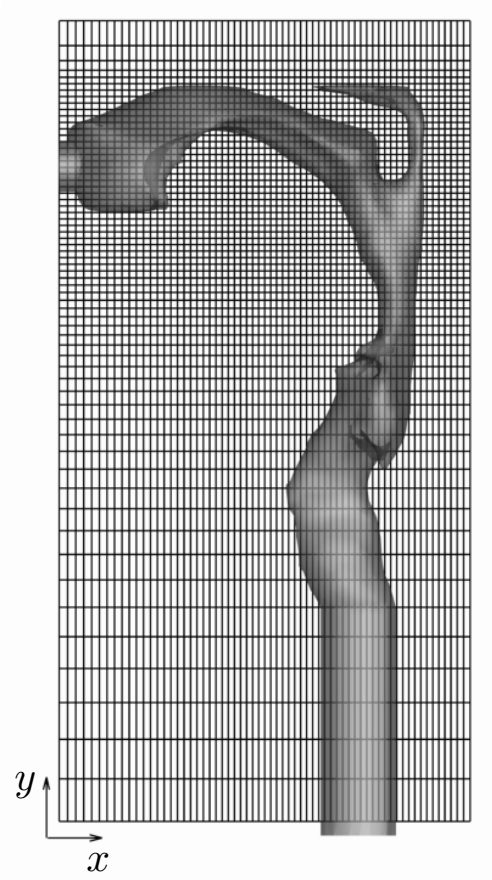

(b)

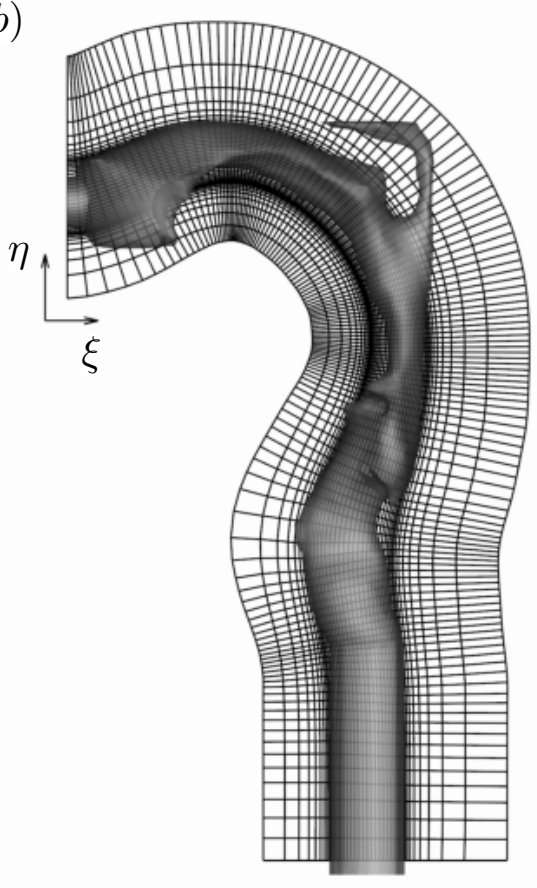

Figure 3: Grids for model S1a. (a) Cartesian grid. Every eighth grid line in $x$ and $y$ has been plotted. (b) Curvilinear grid. Every fourth grid line in $\xi$ and eighth grid line in $\eta$. 
(a)

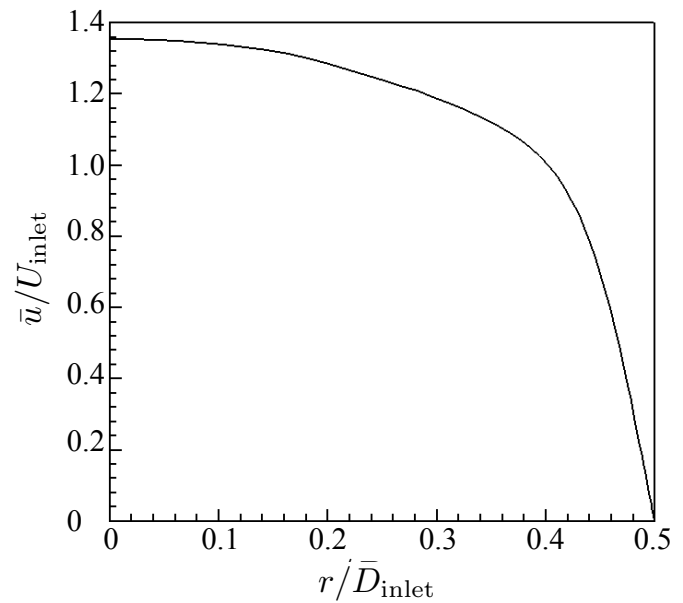

$(b)$

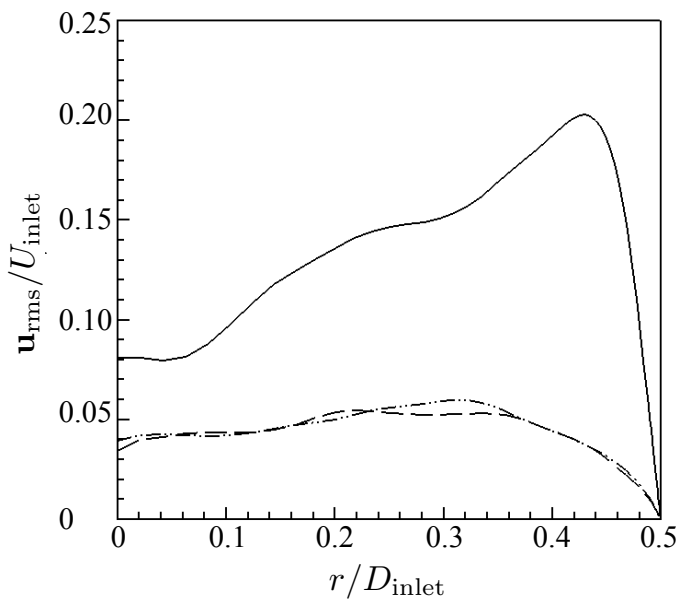

Figure 4: (a) Mean streamwise velocity $\bar{u}$ normalized by the inlet bulk velocity $U_{\text {inlet }}$. $(b)$ Turbulence intensities normalized by the inlet bulk velocity: - , $u_{\mathrm{rms}} ;---, v_{\mathrm{rms}}$; $-\cdots-, w_{\mathrm{rms}}$. 
(a)

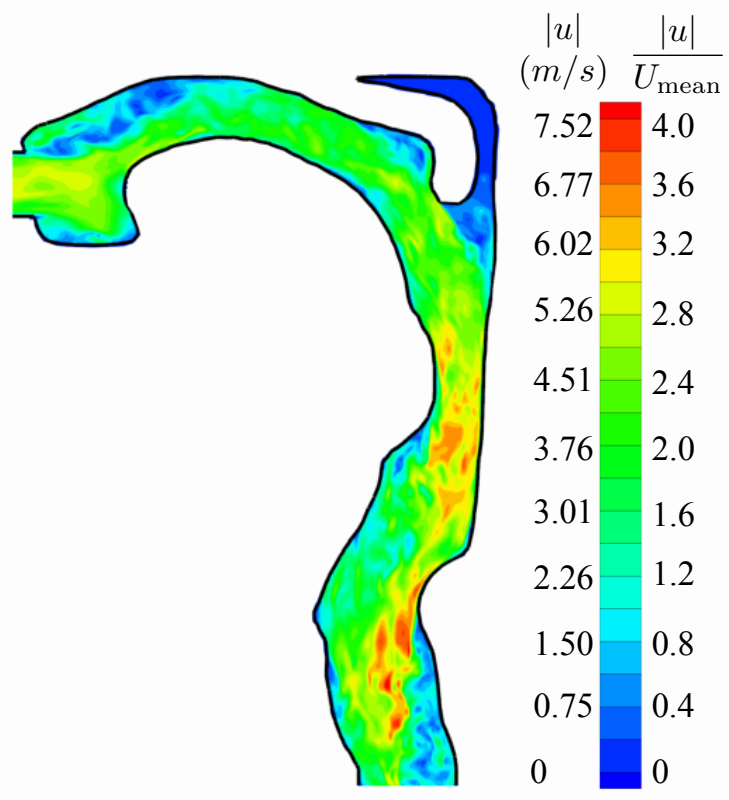

(b)

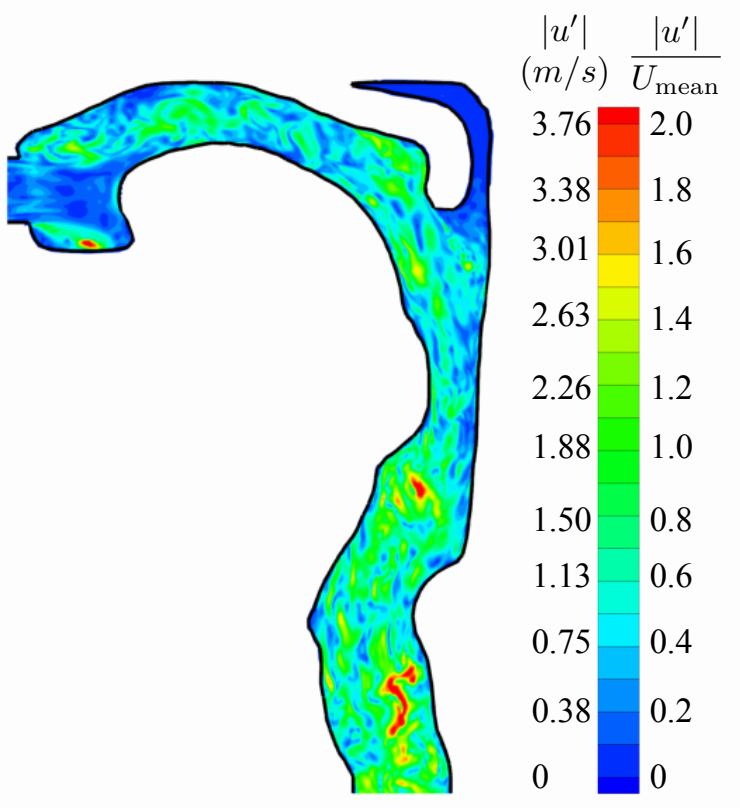

Figure 5: Contours of (a) instantaneous velocity magnitude and $(b)$ fluctuation magnitude in geometry S1a. 
(a)

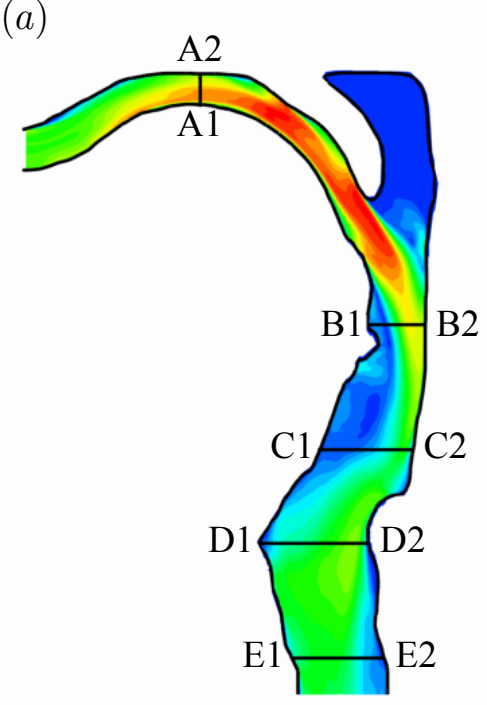

$|\bar{u}| \quad|\bar{u}| \quad(b)$

\begin{tabular}{l|l}
$(\mathrm{m} / \mathrm{s})$ & $\overline{U_{\text {mean }}}$ \\
7.40 & 2.70 \\
6.66 & 2.43 \\
5.92 & 2.16 \\
5.18 & 1.89 \\
\hline 4.44 & 1.62 \\
3.70 & 1.35 \\
\hline 2.96 & 1.08 \\
2.22 & 0.81 \\
\hline 1.48 & 0.54 \\
0.74 & 0.27 \\
0 & 0
\end{tabular}

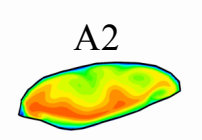

A1

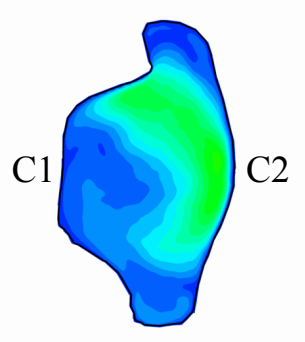

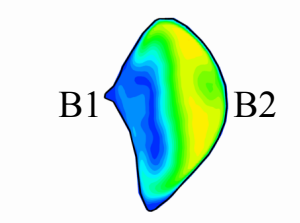
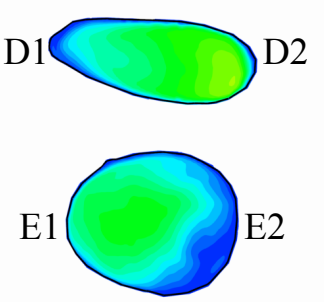

(b)

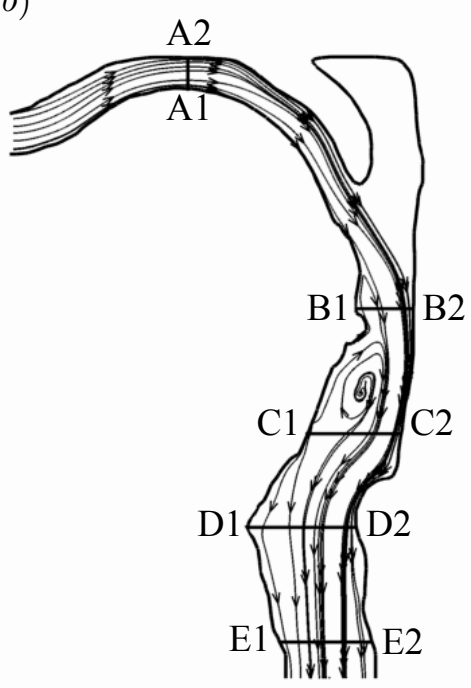

Figure 6: (a) Contours of mean velocity magnitude and (b) 2D streamlines in the central sagittal plane and at various cross-sections of geometry S1b. 
(a)

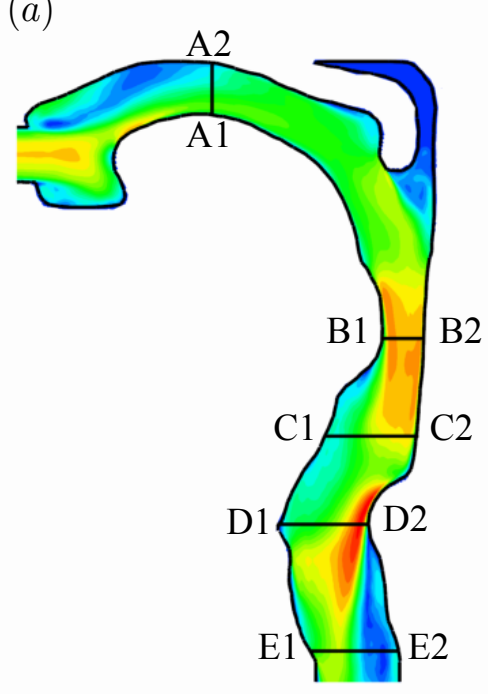

$\underset{(m / s)}{|\bar{u}|} \frac{|\bar{u}|}{U_{\text {mean }}}$

$6.58-3.50$

$5.92 \quad 3.15$

$5.26-2.80$

$4.61-2.45$

$3.95-2.10$

$3.29-1.75$

$2.63 \quad 1.40$

$1.97 \quad 1.05$

$1.32 \quad 0.70$

$\begin{array}{ll}0.66 & 0.35 \\ 0 & 0\end{array}$

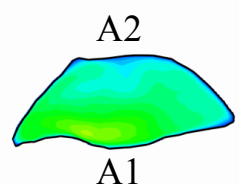

B1
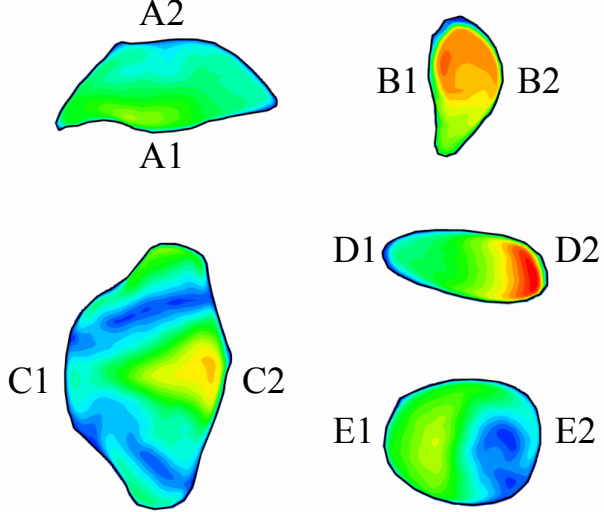

E1

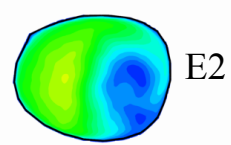

(b)

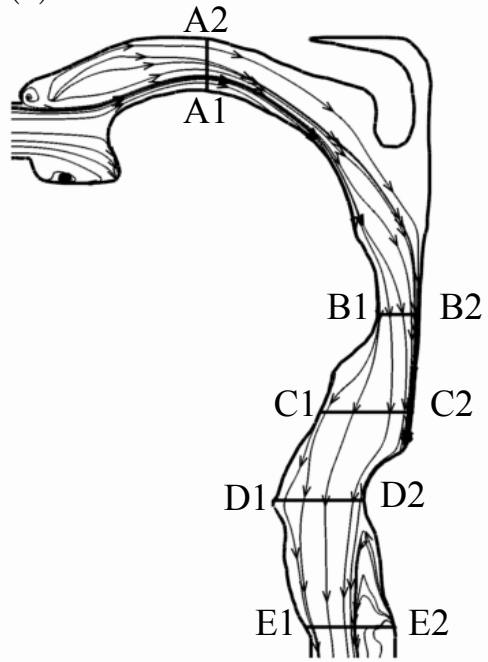

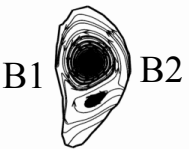

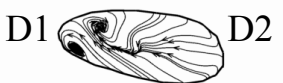

C1

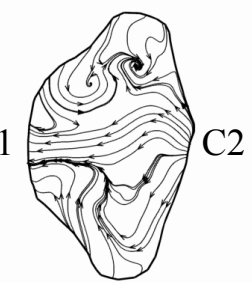

E1

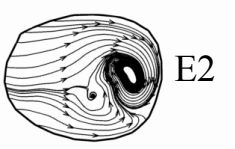

Figure 7: (a) Contours of mean velocity magnitude and (b) 2D streamlines in the central sagittal plane and at various cross-sections of geometry S1a. 
(a)
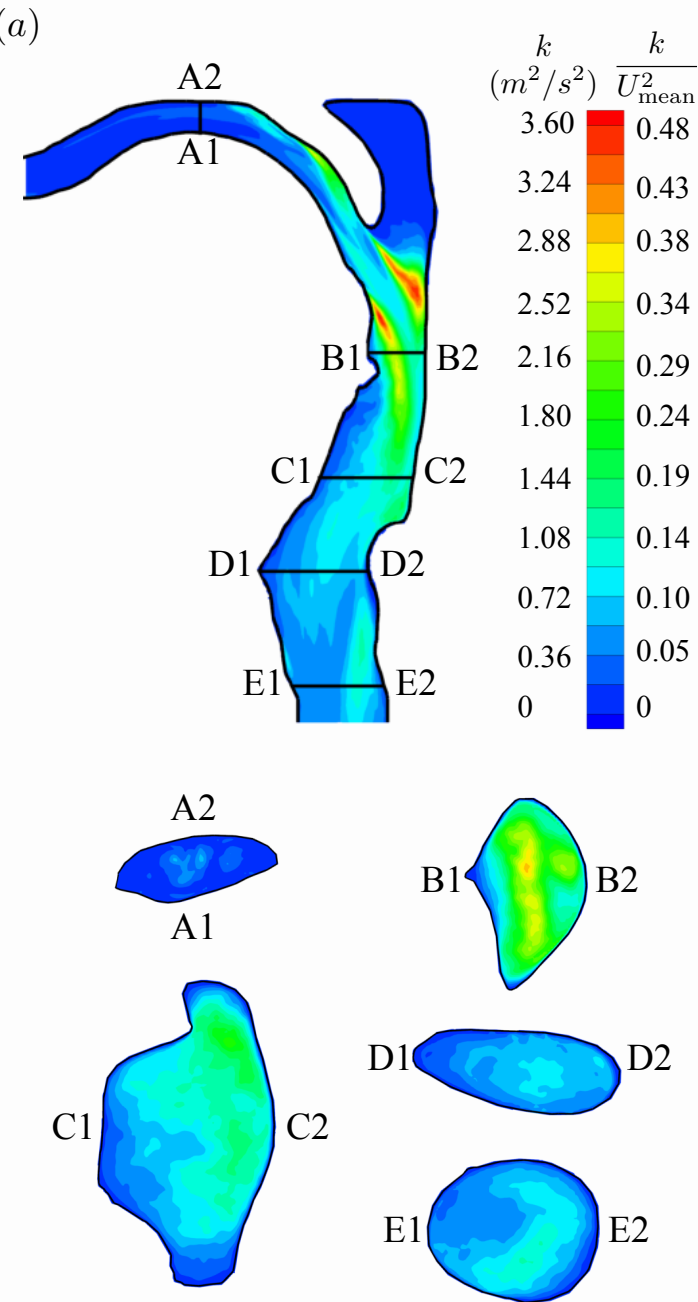
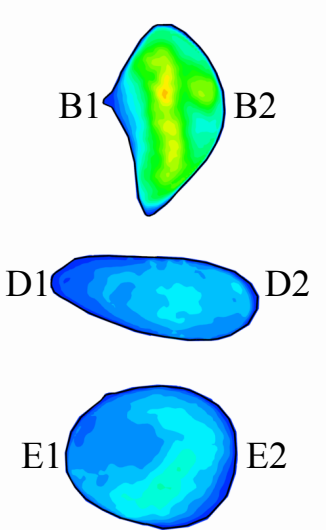

(b)
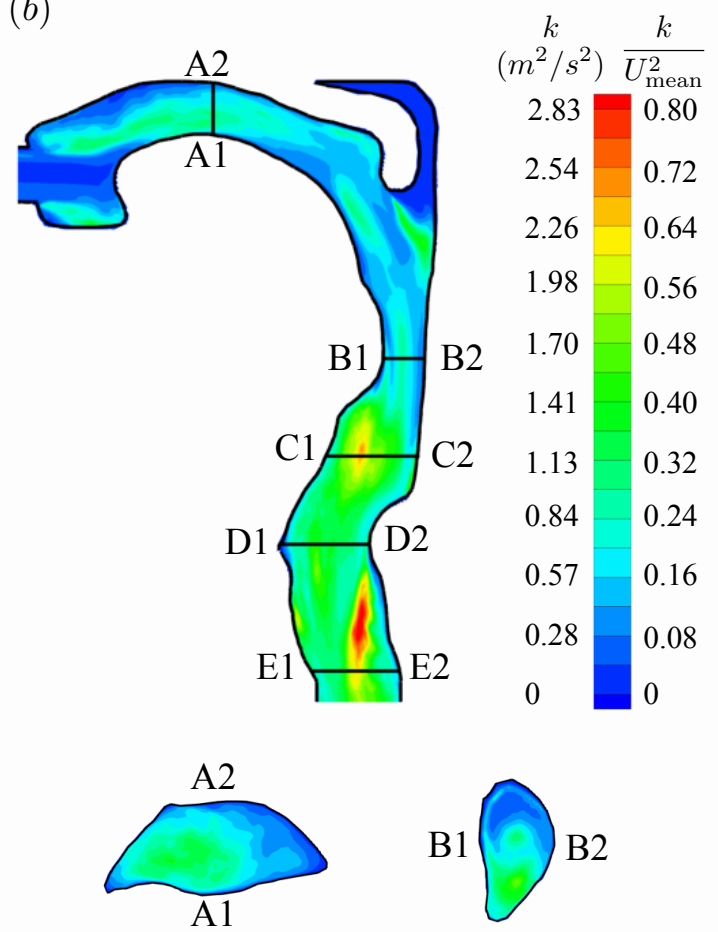

B1
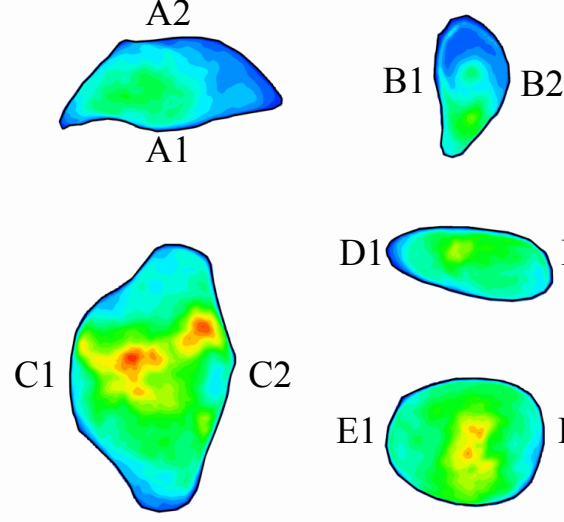

E1

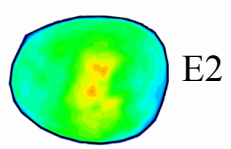

Figure 8: Contours of mean turbulent kinetic energy in the central sagittal plane and at various cross-sections of $(a)$ geometry S1b, and (b) geometry S1a. 
(a)

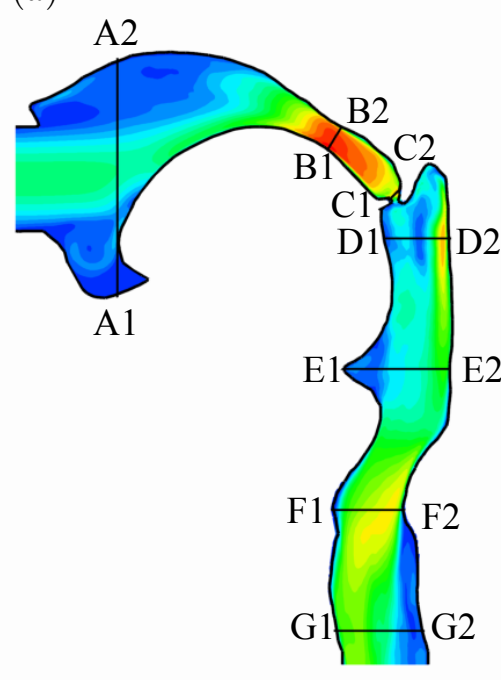

$\underset{(m / s)}{|\bar{u}|} \frac{|\bar{u}|}{U_{\text {mean }}}$ $5.88-5.20$

$5.29 \quad 4.68$

$4.70-4.16$

$4.11-3.64$

$3.53 \quad 3.12$

$2.94 \quad 2.60$

$2.35 \quad 2.08$

$1.76 \quad 1.56$

$1.18 \quad 1.04$

$0.59 \quad 0.52$

0
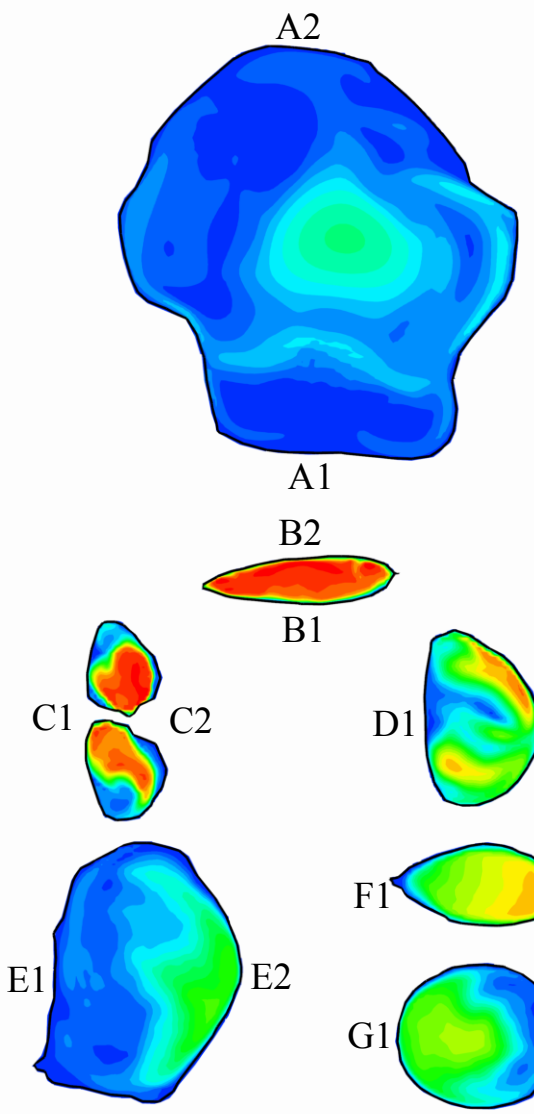

(b)
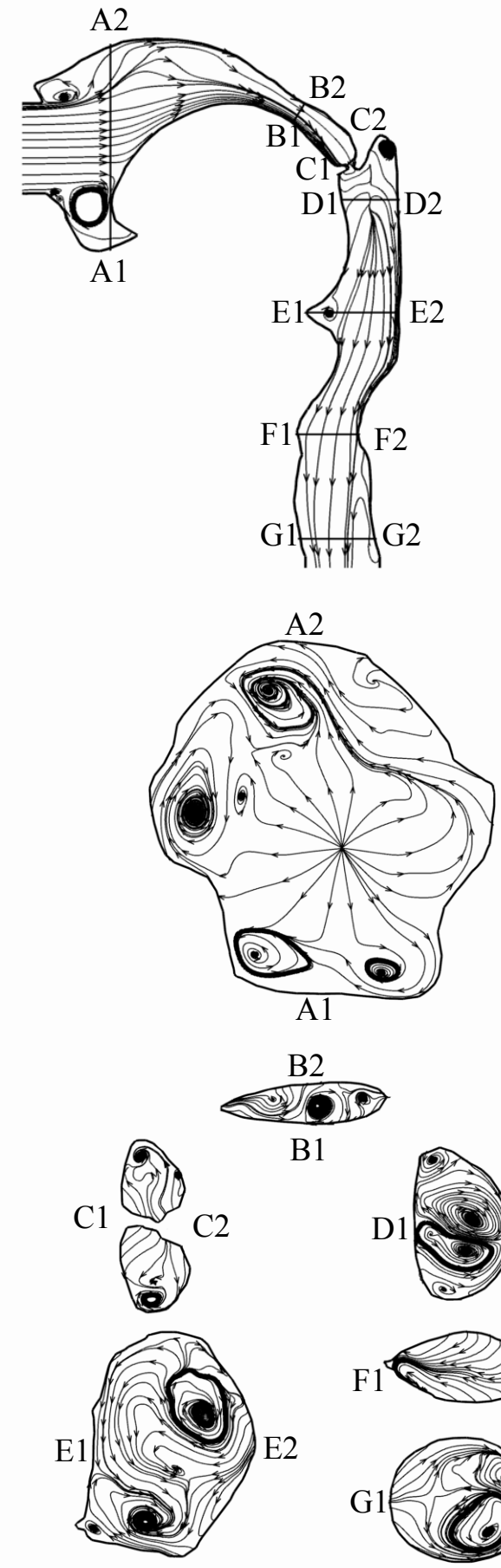

B2

B1
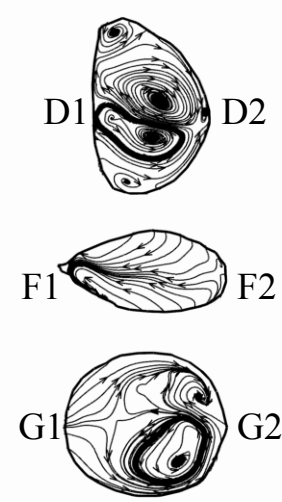

Figure 9: (a) Contours of mean velocity magnitude and (b) 2D streamlines in the central sagittal plane and at various cross-sections of geometry S2. 
(a)
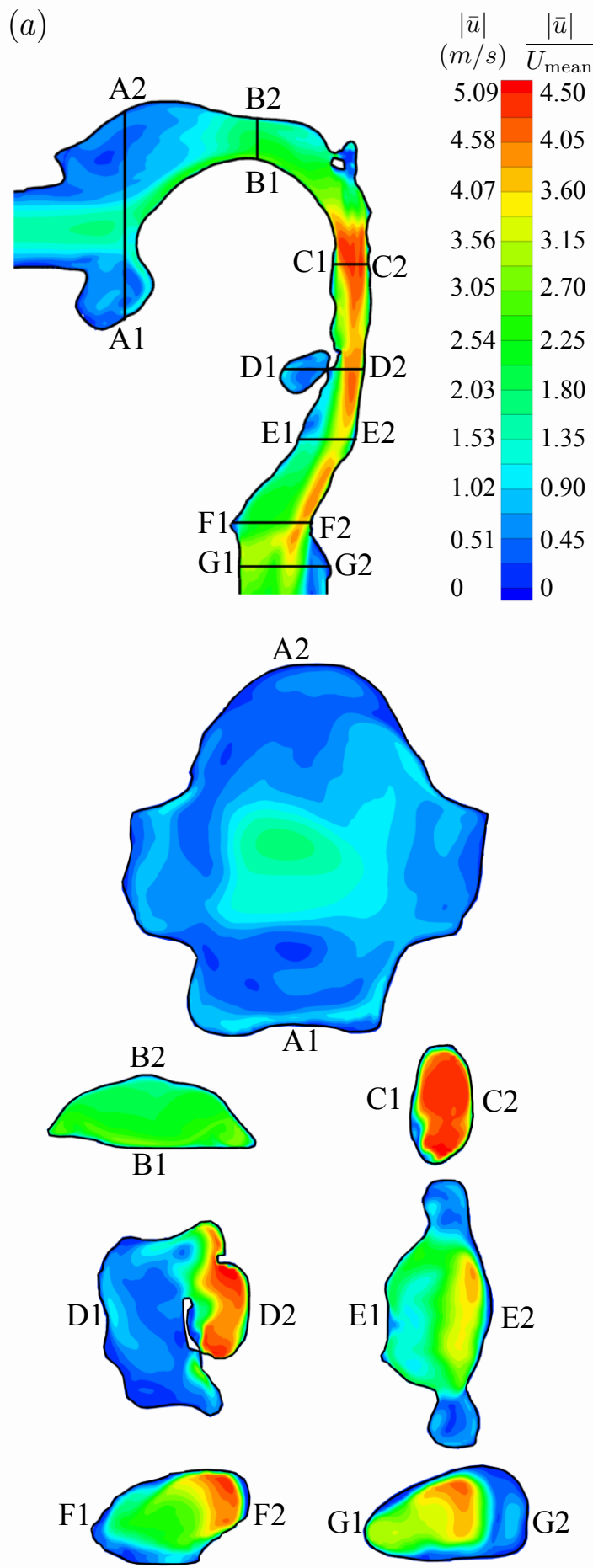

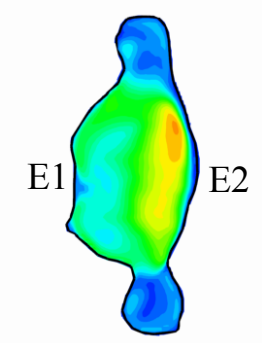

A2
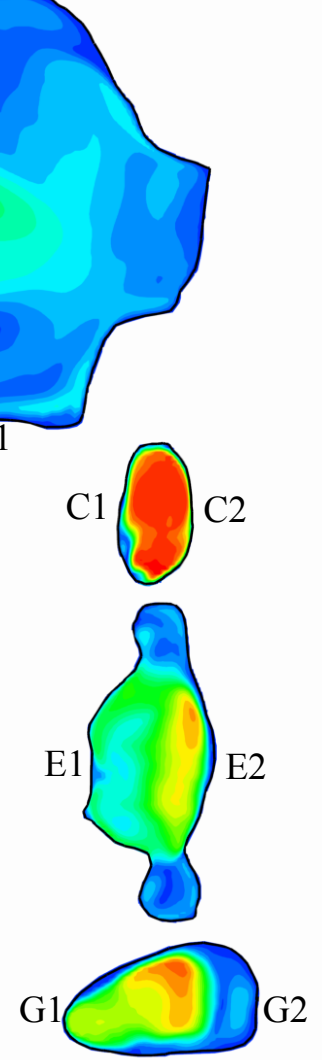

(b)
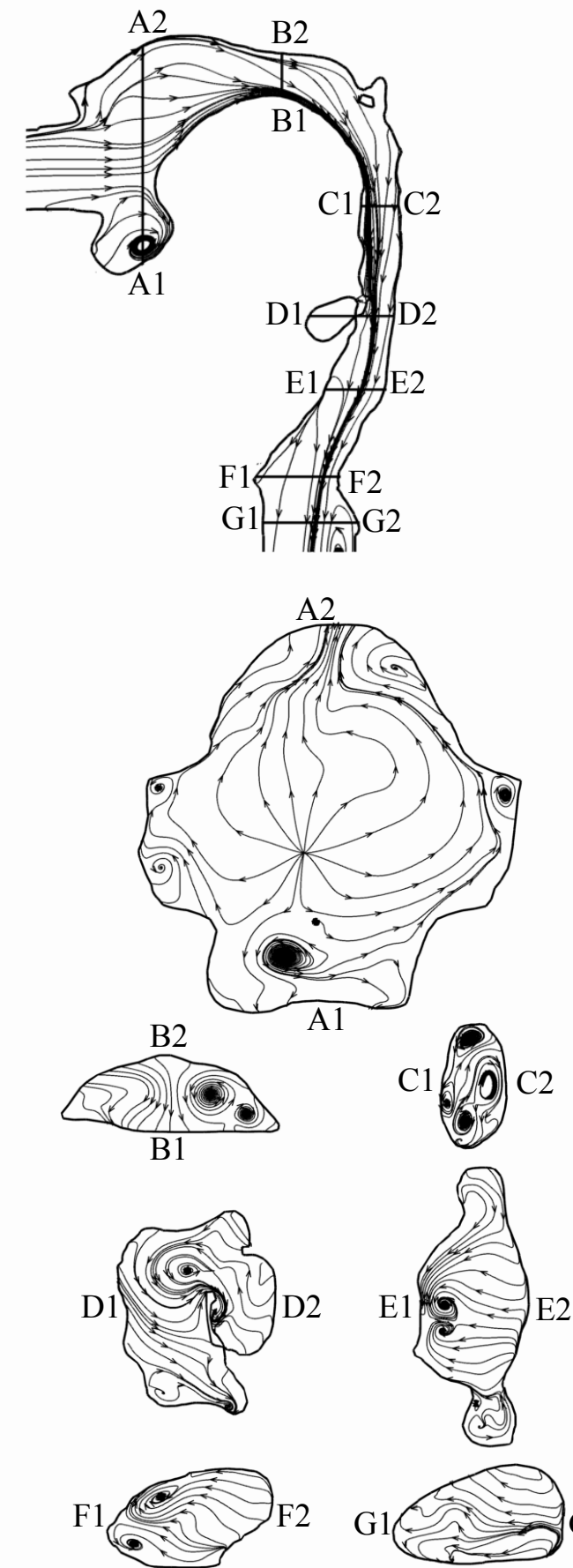

Figure 10: (a) Contours of mean velocity magnitude and (b) 2D streamlines in the central sagittal plane and at various cross-sections of geometry S4. 
(a)

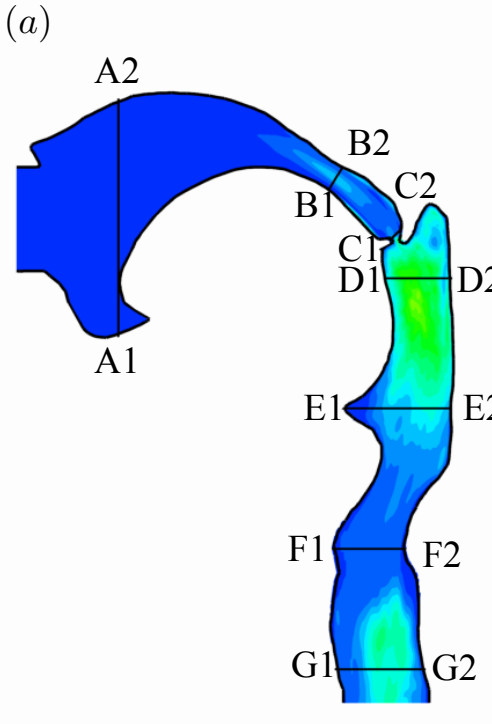

$k$
$\left(m^{2} / s^{2}\right)$$\frac{k}{U_{\text {mean }}^{2}}$

$3.83 \bigcirc 3.00$

3.45

$3.06-2.40$

2.68

2.30

21.92

1.53

1.15

0.77

0.38
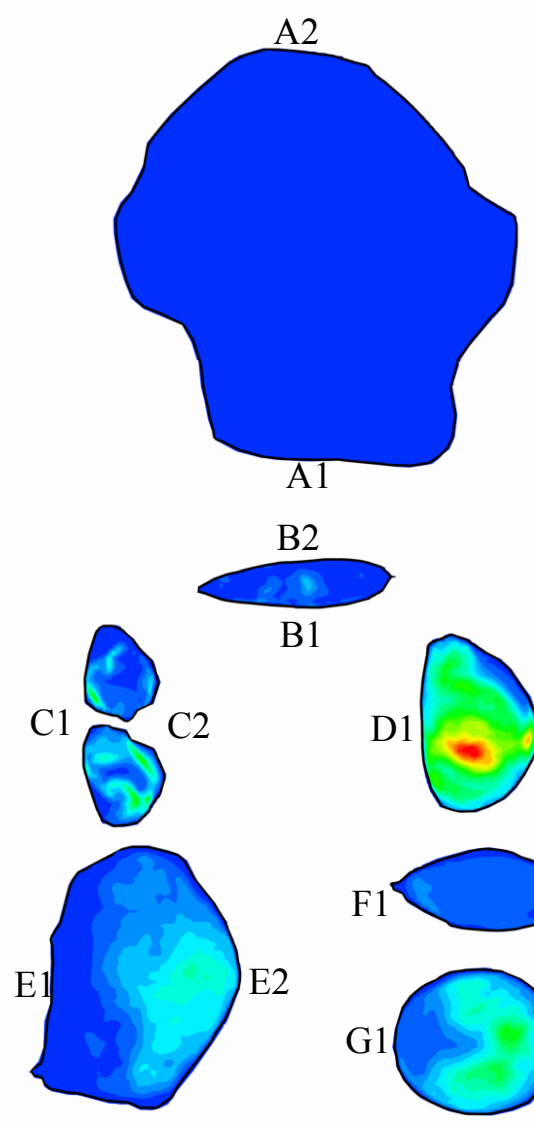

B1

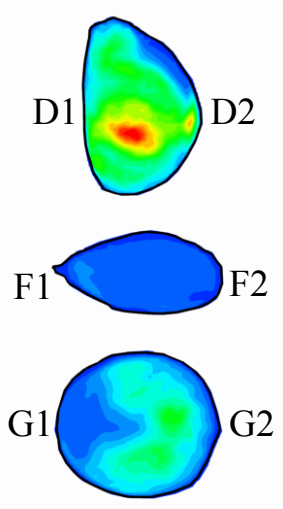

(b)
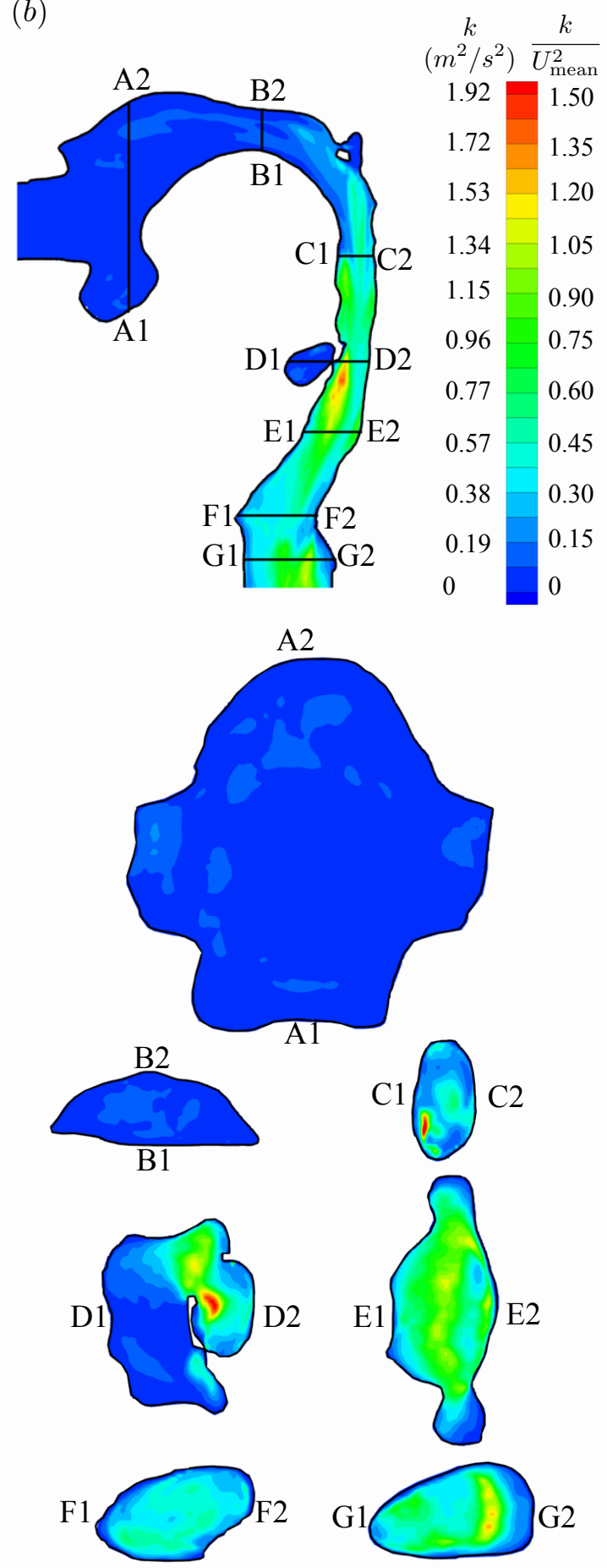

A2
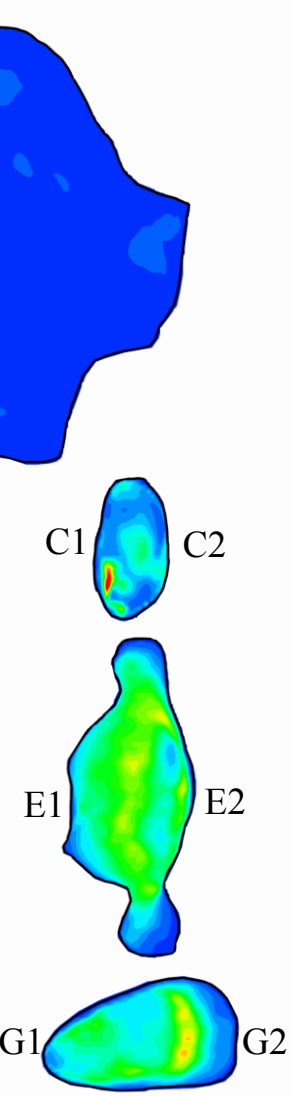

Figure 11: Contours of mean turbulent kinetic energy in the central sagittal plane and at various cross-sections of $(a)$ geometry $\mathrm{S} 2$, and $(b)$ geometry S4. 
Table 1: Dimensions of the mouth and throat geometries.

\begin{tabular}{lrrrr}
\hline Model & \multicolumn{1}{c}{$\mathrm{S} 1 \mathrm{~b}$} & $\mathrm{~S} 1 \mathrm{a}$ & \multicolumn{1}{c}{$\mathrm{S} 2$} & $\mathrm{~S} 4$ \\
\hline$D_{\text {inlet }}(\mathrm{cm})$ & 0.83 & 1.30 & 2.30 & 2.30 \\
Volume $\left(\mathrm{cm}^{3}\right)$ & 38.63 & 51.56 & 81.73 & 84.20 \\
Length $(\mathrm{cm})$ & 18.90 & 19.10 & 18.60 & 19.10 \\
$D_{\text {mean }}(\mathrm{cm})$ & 1.61 & 1.85 & 2.37 & 2.37 \\
\hline
\end{tabular}


Table 2: Flow parameters in the mouth and throat geometries.

\begin{tabular}{lllll}
\hline Model & $\mathrm{S} 1 \mathrm{~b}$ & $\mathrm{~S} 1 \mathrm{a}$ & $\mathrm{S} 2$ & $\mathrm{~S} 4$ \\
\hline$U_{\text {inlet }}(\mathrm{m} / \mathrm{s})$ & 3.92 & 3.77 & 1.20 & 1.20 \\
$U_{\text {mean }}(\mathrm{m} / \mathrm{s})$ & 2.74 & 1.88 & 1.13 & 1.13 \\
$R e_{\text {inlet }}$ & 3292 & 3222 & 1815 & 1815 \\
$R e_{\text {mean }}$ & 2744 & 2262 & 1761 & 1761 \\
\hline
\end{tabular}


Table 3: Deposition in the different regions of the S1a and S1b geometries, given as a percentage of aerosol entering the mouth (Grgic et al., 2004a).

\begin{tabular}{ccrrrrr}
\hline \multirow{2}{*}{$\begin{array}{c}\text { Particle size } \\
(\mu \mathrm{m})\end{array}$} & Geometry & \multicolumn{5}{c}{ Regions } \\
\cline { 3 - 6 } & & Mouth (\%) & Pharynx (\%) & Larynx (\%) & Trachea (\%) & Total $(\%)$ \\
\hline \multirow{2}{*}{3.0} & S1b & $0.56 \pm 0.12$ & $0.62 \pm 0.07$ & $0.38 \pm 0.07$ & $0.17 \pm 0.06$ & $1.73 \pm 0.36$ \\
& S1a & $0.39 \pm 0.04$ & $0.22 \pm 0.01$ & $0.47 \pm 0.06$ & $0.15 \pm 0.01$ & $1.23 \pm 0.12$ \\
\hline \multirow{2}{*}{5.0} & S1b & $11.58 \pm 2.31$ & $11.68 \pm 1.39$ & $10.06 \pm 2.47$ & $1.33 \pm 0.32$ & $34.63 \pm 2.29$ \\
& S1a & $2.86 \pm 0.50$ & $2.14 \pm 0.66$ & $5.71 \pm 1.47$ & $1.10 \pm 0.34$ & $11.81 \pm 2.56$ \\
\hline \multirow{2}{*}{6.5} & S1b & $25.64 \pm 6.66$ & $15.49 \pm 1.77$ & $18.02 \pm 7.59$ & $2.90 \pm 2.25$ & $62.05 \pm 2.63$ \\
& S1a & $24.32 \pm 0.31$ & $10.68 \pm 2.68$ & $20.96 \pm 0.82$ & $6.23 \pm 0.74$ & $62.19 \pm 3.50$ \\
\hline
\end{tabular}


Table 4: Deposition in the different regions of the S2 and S4 geometries, given as a percentage of aerosol entering the mouth (Grgic et al., 2004a).

\begin{tabular}{ccllllc}
\hline \multirow{2}{*}{$\begin{array}{c}\text { Particle size } \\
(\mu \mathrm{m})\end{array}$} & Geometry & \multicolumn{5}{c}{ Regions } \\
\cline { 3 - 7 } 3.0 & & Mouth (\%) & Pharynx (\%) & Larynx (\%) & Trachea (\%) & Total $(\%)$ \\
\hline & $\mathrm{S} 2$ & & & & 0 \\
& $\mathrm{~S} 4$ & 0.49 & 0.67 & 0.33 & 0.13 & 1.62 \\
\hline \multirow{2}{*}{5.0} & $\mathrm{~S} 2$ & $0.93 \pm 0.31$ & $0.30 \pm 0.08$ & $0.33 \pm 0.02$ & $0.33 \pm 0.10$ & $1.89 \pm 0.39$ \\
& $\mathrm{~S} 4$ & 1.31 & 1.85 & 1.49 & 0.63 & 5.28 \\
\hline \multirow{2}{*}{6.5} & $\mathrm{~S} 2$ & $0.66 \pm 0.72$ & $0.87 \pm 0.65$ & $1.73 \pm 0.15$ & $2.98 \pm 0.18$ & $6.24 \pm 1.68$ \\
& $\mathrm{~S} 4$ & 2.46 & 6.09 & 3.99 & 1.44 & 13.98 \\
\hline
\end{tabular}


- Direct numerical simulations are performed in realistic mouth-throat geometries.

- Transition to turbulence occurs even if the inflow is laminar.

- Geometric variation has a large effect on both mean flow and turbulent fluctuations.

- The power-law dependence of deposition on Re is demonstrated analytically.

- Both impaction and dispersion are significant mechanisms of extrathoracic deposition. 